TRANSACTIONS OF THE

AMERICAN MATHEMATICAL SOCIETY

Volume 355, Number 11, Pages 4595-4638

S 0002-9947(03)03319-1

Article electronically published on July 8, 2003

\title{
OPEN 3-MANIFOLDS WHOSE FUNDAMENTAL GROUPS HAVE INFINITE CENTER, AND A TORUS THEOREM FOR 3-ORBIFOLDS
}

\author{
SYLVAIN MAILLOT
}

\begin{abstract}
Our main result is a characterization of open Seifert fibered 3manifolds in terms of the fundamental group and large-scale geometric properties of a triangulation. As an application, we extend the Seifert Fiber Space Theorem and the Torus Theorem to a class of 3-orbifolds.
\end{abstract}

\section{INTRODUCTION}

Let $W$ be an orientable, irreducible 3 -manifold such that $\pi_{1} W$ has infinite center. If $W$ is compact, then the Seifert Fiber Space Theorem [39], 29], [26], 37], 13], [7] asserts that $W$ is a Seifert fiber space. If $W$ is noncompact, this need not be the case: for instance, P. Scott and T. Tucker [30] have constructed examples of fake open solid tori, i.e., open orientable irreducible 3 -manifolds $W$ such that $\pi_{1} W$ is infinite cyclic and yet $W$ is not homeomorphic to $S^{1} \times \mathbf{R}^{2}$. It is easy to see that such a manifold cannot be Seifert fibered.

In this article, we prove that an open, orientable, irreducible 3-manifold whose fundamental group has infinite center is Seifert fibered if it admits a triangulation satisfying some large scale geometric properties. To define these properties formally, we need some terminology: let $W$ be a 3-manifold and let $\mathcal{T}$ be a triangulation of $W$. We say that $\mathcal{T}$ has bounded geometry if there is a uniform bound on the number of simplices in the star of a vertex. Let $i \in\{1,2,3\}$ and let $P$ be an $i$-dimensional simplicial complex. Recall that a map $f: P \rightarrow W$ is called combinatorial if it has image in the $i$-skeleton and maps each $i$-simplex homeomorphically onto some $i$ simplex of $\mathcal{T}$. Thus the number of $i$-simplices of $P$ can be thought of as the number of $i$-simplices of $f(P)$ "counted with multiplicities", and is called the $i$-dimensional volume of the map $f$ and denoted by $\operatorname{Vol}_{i}(f)$. We use the words length and area as synonyms for 1-dimensional and 2-dimensional volume, respectively.

Definition. Let $(W, \mathcal{T})$ be a triangulated manifold and let $a$ be a central element of $\pi_{1} W$. We say that $a$ is uniformly representable if there exists a constant $C_{0} \geq 0$ such that for each vertex $x$ of $\mathcal{T}$, there is a combinatorial loop based at $x$, of length at most $C_{0}$ and representing $a \in \pi_{1} W$.

Definition. Let $(W, \mathcal{T})$ be a triangulated manifold. We say that $(W, \mathcal{T})$ has uniform isoperimetric profile if there exists a function $C_{1}: \mathbf{N} \rightarrow \mathbf{N}$ such that every

Received by the editors September 28, 2001 and, in revised form, November 25, 2002.

2000 Mathematics Subject Classification. Primary 57N10.

(C)2003 American Mathematical Society 
null-homotopic combinatorial map $f: S^{1} \rightarrow W$ can be extended to a combinatorial map $\bar{f}: D^{2} \rightarrow W$ such that $\operatorname{Vol}_{2}(\bar{f}) \leq C_{1}\left(\operatorname{Vol}_{1}(f)\right)$.

Note that those two properties hold, in particular, for any triangulated manifold that admits a cocompact simplicial group action (see Section 9).

Implicit in the above definitions is the idea that a triangulation of a manifold induces a kind of metric structure on $W$, so that it makes sense to speak of quasiisometries between such spaces. Precise definitions are given in Section 2.

Theorem 1.1. Let $W$ be an open, orientable, irreducible 3-manifold. Let $\mathcal{T}$ be a triangulation of bounded geometry of $W$ such that $(W, \mathcal{T})$ has uniform isoperimetric profile. Let a be a uniformly representable central element of infinite order in $\pi_{1} W$. Then the following properties hold:

(i) $W$ admits a Seifert fibration such that a is a power of the element of $\pi_{1} W$ represented by a regular fiber.

(ii) If the subgroup generated by a is maximal among infinite cyclic central subgroups, then there exists a Seifert fibration $p: W \rightarrow B$ and a complete Riemannian metric on $B$ such that the projection map $p$ is a quasi-isometry.

In an unfortunately still unpublished manuscript, G. Mess [26] proved that if $M$ is a closed, orientable, irreducible manifold and $W$ is the infinite cyclic (regular) cover corresponding to some central infinite-order element of $\pi_{1} M$, then $W$ is homeomorphic to $S^{1} \times \mathbf{R}^{2}$, and if $h$ is any Riemannian metric on $W$ invariant under covering transformations, then $(W, h)$ is quasi-isometric to some complete Riemannian plane. Theorem 1.1 can be viewed as a generalization of that result.

Next we discuss some applications. Our aim is to generalize the Seifert Fiber Space Theorem in the context of 3-orbifolds. Theorem 1.1 together with the same arguments as in the case of manifolds and the main theorem of [23] give the result for good 3-orbifolds, i.e., 3-orbifolds that admit a manifold covering. To deal with possibly bad orbifolds, we need a version of Theorem 1.1 where $W$ is not assumed to be irreducible, but only aspherical. The statement of the theorem (given in Section 8 as Theorem 8.1) is fairly technical; so in this introduction we only state the following application.

Theorem 1.2. Let $W$ be an open, orientable 3 -manifold such that $\pi_{1} W$ is infinite cyclic. Let $\Gamma$ be a finitely generated group acting smoothly, properly discontinuously and cocompactly on $W$. Then $\Gamma$ is a virtual closed surface group.

(Recall that a virtual closed surface group is a group that contains a finite index subgroup isomorphic to the fundamental group of some closed surface, not the sphere or projective plane, or equivalently a group that is an extension of a finite group by an infinite closed 2-orbifold group.)

An immediate corollary of Theorem 1.2 is that if $\mathcal{O}$ is a closed, orientable 3orbifold whose fundamental group has a normal infinite cyclic subgroup $Z$ of infinite index, then the quotient of $\pi_{1} \mathcal{O}$ by $Z$ is a virtual closed surface group. This implies that $\pi_{1} \mathcal{O}$ is isomorphic to the fundamental group of some Seifert fibered orbifold. To go on, we have to prove that there are no fake Seifert fibered orbifolds with infinite fundamental group. We shall do this under the hypothesis that $\mathcal{O}$ does not 
contain an incompressible turnover (i.e., sphere with three singular points). Thus we obtain the following generalization of the Seifert Fiber Space Theorem:

Theorem 1.3. Let $\mathcal{O}$ be a closed, orientable, irreducible 3-orbifold without incompressible turnovers. If $\pi_{1} \mathcal{O}$ has an infinite cyclic normal subgroup, then $\mathcal{O}$ is Seifert-fibered.

We complete the work by proving an orbifold version of the Torus Theorem:

Theorem 1.4 (Torus Theorem). Let $\mathcal{O}$ be a closed, orientable, irreducible 3-orbifold without incompressible turnovers. If $\pi_{1} \mathcal{O}$ has a subgroup isomorphic to $\mathbf{Z}^{2}$, then one of the following holds:

(i) $\mathcal{O}$ contains an incompressible Euclidean 2-suborbifold, or

(ii) $\mathcal{O}$ is Euclidean or Seifert-fibered.

We state all theorems on orbifolds in the closed case. Presumably, the techniques of this paper can be extended to deal with compact orbifolds with boundary. For orbifolds with nonempty singular locus, Theorems 1.3 and1.4 are also consequences of Thurston's Orbifold Theorem, whose proof has been recently completed [1, [2], 3], 8], 35, 36. However, the proofs given here are much simpler, and more natural since we do not distinguish between the manifold and the orbifold case.

This article is organized as follows. In Section 2 we define the canonical quasimetric associated to a covering of a space by compact subsets. In particular, this allows us to define quasi-isometries between triangulated 3-manifolds.

In Section 3, we introduce more terminology, which enables us to state three crucial propositions which together imply Theorem [1.1] Proposition 3.1 is a restatement of part (i) of Theorem 1.1, Proposition 3.2 asserts the existence of an $M$ splitting, i.e., a decomposition of $W$ by tori and annuli into Seifert fibered pieces of uniformly bounded size; Proposition 3.3 states that the existence of an M-splitting implies the conclusion of part (ii) of Theorem 1.1

Sections 47 are devoted to the proofs of these three propositions. Section 4 is a short section containing background material on 3-manifold topology. In Section 5 we develop the notions of uniform asphericity and of a cyclic homotopy. We then use this to prove Proposition 3.1 and prepare the proof of Proposition 3.2.

Section 6 is the heart of the paper: using the material of Section 5 Proposition 3.2 is proven, making intensive use of PL minimal surfaces, which are reviewed in the Appendix. In Section 7, we use combinatorial arguments and some more 3-manifold topology to prove Proposition 3.3

In Section 8 , we deal with aspherical open 3-manifolds that are not assumed to be irreducible. The main result there is Theorem 8.1, which is needed for Theorem 1.2 In Section 9 we turn to 3-manifolds admitting cocompact group actions and prove Theorem 1.2. Section 10 is devoted to the applications to orbifolds.

We end the introduction by some general notation and conventions. We let $I$ denote the unit interval $[0,1] \subset \mathbf{R}, D^{n}$ the $n$-dimensional disc, $S^{n}$ the $n$-dimensional sphere and $T^{n}$ the $n$-dimensional torus.

All homology groups have coefficients in $\mathbf{Q}$.

When $f: X \rightarrow Y$ is a map between topological spaces, $f_{*}$ denotes indifferently any homomorphism induced by $f$, either between fundamental groups or homology groups.

In a 3-manifold context, submanifold means codimension-zero submanifold.

The $i$-skeleton of a triangulated manifold $(W, \mathcal{T})$ is denoted by $\mathcal{T}^{(i)}$. 


\section{QuASIMETRICS DEFINED BY COMPACT COVERS}

\subsection{Quasimetric spaces.}

Definition ([5]). Let $X$ be a set and let $C \geq 0$ be a constant. A function $d$ : $X^{2} \rightarrow[0,+\infty)$ is a $C$-quasimetric (or simply a quasimetric) if it has the following properties:

(i) $d(x, x)=0$ for all $x \in X$;

(ii) $d(x, y)=d(y, x)$ for all $x, y \in X$;

(iii) $d(x, z) \leq d(x, y)+d(y, z)+C$ for all $x, y, z \in X$.

We call $(X, d)$ a quasimetric space.

Next we introduce some terminology for quasimetric spaces, most of which extends standard terminology for metric spaces. Let $(X, d)$ be a quasimetric space. Given $x \in X$ and $R \geq 0$, the ball of center $x$ and radius $R$, written $B_{d}(x, R)$, is the set of $y \in X$ such that $d(x, y) \leq R$. Given $A \subset X$, the $R$-neighborhood of $A$ is the set $N_{d}(A, R)=\bigcup_{x \in A} B_{d}(x, R)$. We say that $A$ is $(R$ - $)$ quasidense in $X$ if $N_{d}(A, R)=X$. The $d$-diameter of $A$ (or simply diameter if the quasimetric is understood) is $\operatorname{diam}_{d} A:=\sup _{x, y \in A} d(x, y) \in[0,+\infty]$. We say that $A$ is bounded if $\operatorname{diam}_{d} A<+\infty$. Indices will often be dropped when there is no ambiguity.

Definition. Let $\left(X_{1}, d_{1}\right)$ and $\left(X_{2}, d_{2}\right)$ be quasimetric spaces. We say that a map $f: X_{1} \rightarrow X_{2}$ is a quasi-isometry if there exist constants $\lambda \geq 1$ and $C \geq 0$ such that:

(i) The inequalities

$$
\lambda^{-1} d_{1}\left(x, x^{\prime}\right)-C \leq d_{2}\left(f(x), f\left(x^{\prime}\right)\right) \leq \lambda d_{1}\left(x, x^{\prime}\right)+C
$$

hold for any $x, x^{\prime} \in X_{1}$.

(ii) The image of $f$ is $C$-quasidense in $X_{2}$.

It can be shown that if there is a quasi-isometry $f: X_{1} \rightarrow X_{2}$, then there is a quasi-isometry $g: X_{2} \rightarrow X_{1}$ such that $f \circ g$ (resp. $g \circ f$ ) is a bounded distance from the identity of $X_{2}$ (resp. $X_{1}$ ). In that case, we say that $X_{1}$ and $X_{2}$ are quasiisometric. Being quasi-isometric is an equivalence relation between quasimetric spaces.

2.2. Compact covers. Let $X$ be a path-connected topological space and $\mathcal{K}$ a family of path-connected compact subsets of $X$ such that $X=\bigcup \mathcal{K}$. We call $\mathcal{K}$ a compact cover. We always suppose that $\mathcal{K}$ is locally finite, which means that no compact $A \subset X$ can meet infinitely many elements of $\mathcal{K}$.

Define the size of a subset $A \subset X$, denoted $\operatorname{size}_{\mathcal{K}}(A)$, as the minimal cardinal of a subfamily $\mathcal{K}^{\prime}$ of $\mathcal{K}$ such that $A \subset \cup \mathcal{K}^{\prime}$. This number may be infinite in general, but by local finiteness, a compact subset has always finite size.

Let $x, y$ be points of $X$. A minimizing path between $x$ and $y$ is a continuous path $\alpha:[0,1] \rightarrow X$ such that $\alpha(0)=x, \alpha(1)=y$ and $\alpha$ has minimal size. Such a path always exists because $X$ is path-connected.

Definition. The canonical quasimetric associated to the locally finite compact cover $\mathcal{K}$ is the 1 -quasimetric on $X$ defined by $d_{\mathcal{K}}(x, y)=\operatorname{size}_{\mathcal{K}}(\alpha)-1$ where $\alpha$ is any minimizing path between $x$ and $y$.

The following lemma gives an alternative definition for $d_{\mathcal{K}}$, which will also be used in this paper. 
Lemma 2.1. Let $x, y$ be points of $X$. Then $d_{\mathcal{K}}(x, y)$ is the minimal number $n$ such that there exists a finite sequence $K_{0}, \ldots, K_{n}$ of elements of $\mathcal{K}$ such that $x \in K_{0}$, $y \in K_{n}$ and $K_{i} \cap K_{i+1} \neq \emptyset$ for all $0 \leq i \leq n-1$.

Proof. Consider a finite sequence $K_{0}, \ldots, K_{n}$ as above, with $n$ minimal. Define a finite sequence $x_{0}, \ldots, x_{n+1}$ by setting $x_{0}=x, x_{n+1}=y$ and choosing for $x_{i}$ any point of $K_{i-1} \cap K_{i}$ for $1 \leq i \leq n$. Since all elements of $\mathcal{K}$ are path-connected, there is a path $\alpha_{i} \subset K_{i}$ connecting $x_{i}$ to $x_{i+1}$ for all $0 \leq i \leq n$. The concatenation $\alpha_{0} \cup \cdots \cup \alpha_{n}$ is a path of size at most $n+1$ between $x$ and $y$. This proves that $d_{\mathcal{K}}(x, y) \leq n$.

For the reverse inequality, let $\alpha$ be a minimizing path between $x$ and $y$. Let $L_{0}, \ldots, L_{m}$ be elements of $\mathcal{K}$ such that $\alpha$ lies in $L_{0} \cup \cdots \cup L_{m}$, with $m=d_{\mathcal{K}}(x, y)=$ $\operatorname{size}(\alpha)-1$. Assume that $x \in L_{0}$ and $y \in L_{m}$. Consider the abstract graph $\mathcal{G}$ with $m+1$ vertices, numbered from 0 to $m$, where there is an edge between $i$ and $j$ iff $L_{i} \cap L_{j} \neq \emptyset$. We will show that there is an edge path in $\mathcal{G}$ connecting 0 to $m$, which implies the existence of a sequence $K_{0}, \ldots, K_{n}$ as above with $n \leq m$, completing the proof of the lemma.

If $m=0$, there is nothing to prove. Otherwise write

$$
\alpha=\left(\alpha \cap L_{0}\right) \cup\left(\alpha \cap \bigcup_{i>0} L_{i}\right) .
$$

This gives $\alpha$ as the union of two nonempty closed subsets. Since $\alpha$ is connected, this union cannot be disjoint. Hence some $L_{i}$ meets $L_{0}$, i.e., there is an edge in $\mathcal{G}$ between 0 and some vertex $i>0$. Again we can write $\alpha$ as a union of two nonempty closed subsets:

$$
\alpha=\left(\alpha \cap\left(L_{0} \cup L_{i}\right)\right) \cup\left(\alpha \cap \bigcup_{j \notin\{0, i\}} L_{j}\right) .
$$

This shows that there is an edge between some vertex $j \notin\{0, i\}$ and either 0 or $i$. Repeating this process, we eventually reach $m$ and we obtain an edge path between 0 and $m$ in $\mathcal{G}$.

Definition. A locally finite compact cover $\mathcal{K}$ of a space $X$ has bounded geometry if there is a constant $\nu \in \mathbf{N}$ such that any $K \in \mathcal{K}$ meets at most $\nu$ elements of $\mathcal{K}$. The smallest such $\nu$ is called the local complexity of $\mathcal{K}$ and denoted by $\nu(\mathcal{K})$.

Lemma 2.2. Let $A \subset X$ be a bounded, path-connected subset. Then we have $\operatorname{diam} A \leq \operatorname{size}(A)$. If $\mathcal{K}$ has bounded geometry, then we also have $\operatorname{size}(A) \leq$ $\nu(\mathcal{K})^{\operatorname{diam} A+1}$.

Proof. Let $x, y$ be points of $A$. By hypothesis, there is a path in $A$ connecting $x$ and $y$. The size of this path is at most the size of $A$. This proves that for all $x, y \in A$, $d_{\mathcal{K}}(x, y) \leq \operatorname{size}(A)$ and the first inequality follows.

For the second inequality, pick a point $x \in A$ and observe that $A \subset B(x, \operatorname{diam} A)$; so $\operatorname{size}(A) \leq \operatorname{size}(B(x, \operatorname{diam} A))$. This reduces the problem to the inequality

$$
\operatorname{size}(B(x, r)) \leq \nu(\mathcal{K})^{r+1} \quad \forall(x, r) \in X \times \mathbf{N},
$$

which we shall prove by induction on $r$.

The ball of radius 0 around $x$ is just the union of the elements of $\mathcal{K}$ containing $x$; so inequality (1) for $r=0$ is just the definition of $\nu(\mathcal{K})$. The ball of radius $r+1$ is obtained from the ball of radius $r$ by adding at most $r(\nu(\mathcal{K})-1)$ elements of $\mathcal{K}$. Hence $\operatorname{size}(B(x, r+1)) \leq \nu(\mathcal{K}) \operatorname{size}(B(x, r))$ and inequality (1) is proven. 
Definition. Let $X$ be a path-connected space. Let $\mathcal{K}, \mathcal{K}^{\prime}$ be locally finite compact covers of $X$. Assume that both $\mathcal{K}$ and $\mathcal{K}^{\prime}$ have bounded geometry. We say that $\mathcal{K}^{\prime}$ has bounded geometry with respect to $\mathcal{K}^{\prime}$ if there is a constant $\nu\left(\mathcal{K}, \mathcal{K}^{\prime}\right)$ such that any $K \in \mathcal{K}^{\prime}$ meets at most $\nu\left(\mathcal{K}, \mathcal{K}^{\prime}\right)$ elements of $\mathcal{K}$. If $\mathcal{K}^{\prime}$ has bounded geometry with respect to $\mathcal{K}$ and $\mathcal{K}$ has bounded geometry with respect to $\mathcal{K}^{\prime}$, then we say that $\mathcal{K}$ and $\mathcal{K}^{\prime}$ are equivalent.

The next lemma is obvious from the definitions.

Lemma 2.3. If $\mathcal{K}$ and $\mathcal{K}^{\prime}$ are equivalent, then the identity map is a quasi-isometry from $\left(X, d_{\mathcal{K}}\right)$ to $\left(X, d_{\mathcal{K}^{\prime}}\right)$.

2.3. The case of triangulated manifolds. The construction of the previous subsection will be used in several contexts, but our most important example is the following: let $M$ be a (connected) PL manifold and $\mathcal{T}$ a triangulation of $M$. Let $\mathcal{K}(\mathcal{T})$ be the collection of all simplices (of any dimension) of $\mathcal{T}$. This is a locally finite covering of $M$ by path-connected compact subsets, and it has bounded geometry if and only if $\mathcal{T}$ has bounded geometry as defined in the introduction, i.e., there is a uniform bound on the number of simplices in the star of a vertex. For brevity, we write $d_{\mathcal{T}}=d_{\mathcal{K}(\mathcal{T})}$. Note that when computing the size of a subset, one may restrict attention to simplices of maximal size. In particular, the size of a 3 -subcomplex is equal to its 3 -dimensional volume as defined in the introduction.

We say that a subdivision $\mathcal{T}^{\prime}$ of $\mathcal{T}$ is uniform if there is a constant $C \in \mathbf{N}$ such that any simplex of $\mathcal{T}$ is subdivided into at most $C$ simplices. The following crucial remark will be used repeatedly in the sequel.

Remark. Let $M$ be a PL manifold, $\mathcal{T}$ a triangulation of $M$ and $\mathcal{T}^{\prime}$ a uniform subdivision of $\mathcal{T}$. Then $\mathcal{K}(\mathcal{T})$ and $\mathcal{K}\left(\mathcal{T}^{\prime}\right)$ are equivalent. Hence by Lemma 2.3, the identity map of $M$ is a quasi-isometry between $\left(M, d_{\mathcal{T}}\right)$ and $\left(M, d_{\mathcal{T}^{\prime}}\right)$.

\section{Statements of technical Results}

Throughout the paper, we work with a 3 -manifold $W$ endowed with a triangulation and a central element of infinite order of $\pi_{1} W$. For convenience, we give a name to such an object.

Definition. A TMC (for "Triangulated Manifold with a Central element") is a triple $(W, \mathcal{T}, a)$ where $W$ is a smooth 3 -manifold, $\mathcal{T}$ is a smooth triangulation of bounded geometry of $W$, and $a$ is a nontorsion central element of $\pi_{1} W$. It is maximal if the infinite cyclic subgroup generated by $a$ is maximal among cyclic central subgroups.

We say that a TMC is orientable, irreducible, etc. when the 3-manifold $W$ has this property. Whenever we speak of a bounded subset of $W$, or of the diameter of such a subset, or of quasi-isometries, the canonical quasimetric $d_{\mathcal{T}}$ is understood.

Definition. Let $(W, \mathcal{T}, a)$ be an open, orientable TMC. Let $V$ be a submanifold of $W$. A compatible Seifert fibration on $V$ is a Seifert fibration of $V$ such that the base orbifold is orientable and $a$ is a power of the element of $\pi_{1} W$ represented by the generic fiber.

Definition. Let $(W, \mathcal{T}, a)$ be an open, orientable, maximal TMC. An $M$-splitting of $(W, \mathcal{T}, a)$ is a collection $\mathcal{M}$ of embedded combinatorial tori and annuli such that: 
(i) For each annulus $A \in \mathcal{M}$, there are two (possibly equal) tori $T_{1}, T_{2} \in \mathcal{M}$ such that $\partial A \subset T_{1} \cup T_{2}$.

(ii) Any two elements of $\mathcal{M}$ are disjoint except in the previous situation.

(iii) For each annulus $A \in \mathcal{M}$, the map $\pi_{1} A \rightarrow \pi_{1} W$ is injective and its image is the subgroup generated by $a$.

(iv) Let $V$ be the closure of a component of $W-\cup \mathcal{M}$. Then $V$ is a compact, aspherical submanifold and $H_{2}(\operatorname{Int} V)=0$.

(v) Components of $W-\bigcup \mathcal{M}$ have uniformly bounded diameter.

The proof of Theorem 1.1 breaks up into three propositions:

Proposition 3.1. Let $(W, \mathcal{T}, a)$ be an open, orientable, irreducible TMC. If a is uniformly representable and $(W, \mathcal{T})$ has uniform isoperimetric profile, then $W$ admits a compatible Seifert fibration.

Proposition 3.2. Let $(W, \mathcal{T}, a)$ be an open, orientable, irreducible, maximal TMC. If a is uniformly representable and $(W, \mathcal{T})$ has uniform isoperimetric profile, then there is a uniform subdivision $\mathcal{T}_{2}$ of $\mathcal{T}$ such that $\left(W, \mathcal{T}_{2}, a\right)$ admits an $M$-splitting.

Proposition 3.3. Let $\left(W, \mathcal{T}_{2}, a\right)$ be an open, orientable, irreducible, maximal TMC. If $\left(W, \mathcal{T}_{2}, a\right)$ admits an $M$-splitting, then there is a compatible Seifert fibration on $W$ with base $B$ and projection map $p: W \rightarrow B$ and a complete Riemannian metric on $B$ such that $p$ is a quasi-isometry.

Since replacing $\mathcal{T}$ by the uniform subdivision $\mathcal{T}_{2}$ does not change the quasi-isometry class of the associated quasimetric, it is clear that these three propositions together imply Theorem 1.1

\section{Preliminaries on 3-Manifold topology}

Recall some definitions. A map $f: F \rightarrow M$ of a surface $F$ to a 3-manifold $M$ is proper if $f^{-1}(\partial M)=\partial F$. Two maps $f, g$ are properly homotopic if there is a homotopy $H: F \times I \rightarrow M$ such that $F_{0}=f, F_{1}=g$ and all maps $F_{t}$ are proper. In this paper, all maps and all homotopies are assumed to be proper unless stated explicitly otherwise.

Assume that $F, M$ are orientable and $F$ is not a sphere. We say that $f$ is incompressible if $f_{*}: \pi_{1} F \rightarrow \pi_{1} M$ is injective; otherwise it is compressible. Similarly, $f$ is $\partial$-incompressible if $f_{*}: \pi_{1}(F, \partial F) \rightarrow \pi_{1}(M, \partial M)$ is injective and $\partial$-compressible otherwise. For embedded surfaces, it follows from the Loop Theorem that these definitions are equivalent to those in [16] involving compression discs.

A compact, orientable, irreducible 3-manifold is called a Haken manifold if it contains a compact, orientable, incompressible embedded surface. Every compact, orientable, irreducible 3-manifold with nonempty boundary is Haken [16.

A submanifold $V \subset M$ is incompressible if each component of $\partial V$ is incompressible in $M$ or, equivalently, if there is no compression disc for $\partial V$. By van Kampen's theorem, this implies that the inclusion map $V \rightarrow M$ is $\pi_{1}$-injective.

The following theorem of F. Waldhausen will be one of our main tools in Section 5 .

Theorem 4.1 (Waldhausen 39]). Let $M$ be a Haken manifold. If $\pi_{1} M$ has an infinite cyclic central subgroup $Z$, then $M$ admits a Seifert fibration such that $Z$ is generated by a power of the regular fiber. 
Lemma 4.2. Let $M$ be an irreducible compact Seifert fiber space with nonempty boundary. Then any nonzero element of $H_{2}(M)$ can be represented by a family of pairwise disjoint, embedded, incompressible tori.

Proof. Since $\partial M \neq \emptyset, M$ is a Haken manifold. So any nonzero element of $H_{2}(M)$ can be represented by a family of incompressible closed surfaces. By [38], such surfaces must be vertical tori; so they can be made pairwise disjoint by simple cut and paste operations.

Theorem 4.3 (Jaco-Shalen [18, Johannson [19]). Let $M$ be a Haken manifold. If $f: T^{2} \rightarrow M$ is a singular incompressible torus, then there exists an incompressible submanifold $V \subset M$ that is a Seifert fiber space and a singular torus $g: T^{2} \rightarrow M$ homotopic to $f$ such that $g\left(T^{2}\right)$ lies in $V$.

\section{THE CYCLIC HOMOTOPY AND ITS APPLICATIONS}

\subsection{Uniform asphericity.}

Lemma 5.1. Let $(W, \mathcal{T}, a)$ be an aspherical, uniformly representable TMC. Then there exists a function $C_{2}: \mathbf{N} \rightarrow \mathbf{N}$ such that for any compact $K \subset W$, there is an aspherical compact submanifold $L$ of $W$ such that $K \subset \operatorname{Int} L$ and $\operatorname{diam} L \leq$ $C_{2}(\operatorname{diam} K)$.

Proof. Let $K$ be a compact subset of $W$. Let $Y$ be a regular neighborhood of $N(K, 0)$ obtained by taking the star in the second derived triangulation. If $Y$ is aspherical, then we can set $L=Y$. Otherwise the Sphere Theorem gives an embedded 2-sphere $S$ that does not bound a homotopy ball in $Y$. Since $W$ is aspherical, there is a homotopy ball $B \subset W$ such that $\partial B=S$. At least one component $U$ of $W-\operatorname{Int} Y$ is contained in $B$. Now $\partial U$ is $C_{0}$-quasidense in $U$, because otherwise some point $x$ of $U$ would have the property that any loop based at $x$ of length at most $C_{0}$ is contained in $U$, hence in $B$, contradicting the uniform representability of $a$.

From this, we deduce a bound in terms of $\operatorname{diam} K$ on the diameter of the union of $Y$ and all components of $W-\operatorname{Int} Y$ that lie in homotopy balls. Call $L$ this union. By the Sphere Theorem, it is aspherical; so Lemma 5.1 is proven.

We call uniformly aspherical a triangulated 3-manifold satisfying the conclusion of Lemma 5.1. If, in addition, $W$ is irreducible, then $W$ is called uniformly irreducible.

In the following lemma, the diameter of a map is defined to be the diameter of its image.

Lemma 5.2. Let $(W, \mathcal{T})$ be a uniformly aspherical triangulated 3-manifold. Then for any $i>1$, every continuous map $f: S^{i} \rightarrow W$ can be extended to a continuous map $\bar{f}: D^{i} \rightarrow W$ such that $\operatorname{diam}(\bar{f}) \leq C_{2}(\operatorname{diam}(f))$.

Proof. Let $K$ be the image of $f$. The extension $\bar{f}$ can be found in the aspherical submanifold $L$.

Lemma 5.3. Let $(W, \mathcal{T})$ be a triangulated 3-manifold. Let $P$ be an $i$-dimensional connected simplicial complex (where $1 \leq i \leq 3$ ) and $f: P \rightarrow W$ a combinatorial map. Then $\operatorname{diam}(f(P)) \leq \operatorname{Vol}_{i}(f)$. 
Proof. The $i$-dimensional volume of $f$ is at least the number of $i$-simplices of $f(P)$. Since each $i$-simplex is contained in a 3 -simplex, this number is at least the size of $f(P)$. Now Lemma 2.2 implies that $\operatorname{diam}(f(P)) \leq \operatorname{size}(f(P))$ since $f(P)$ is path-connected.

5.2. Cyclic homotopies. A basepoint in $S^{1}$, denoted by 0 , will be fixed throughout.

Definition. A cyclic homotopy on a $\operatorname{TMC}(W, \mathcal{T}, a)$ is a map $H: W \times S^{1} \rightarrow W$ such that:

(i) $H(\cdot, 0)$ is the identity of $W$.

(ii) For all $x \in W$, the loop $H(x, \cdot)$ represents $a$ (this makes sense without reference to a basepoint because $a$ is central).

Given a point $x \in W$, the loop $H(x, \cdot)$ is called the track of $x$ by $H$.

The diameter of $H$, denoted by diam $H$, is the supremum of the diameters of its tracks.

We say that $H$ is bounded if $\operatorname{diam} H$ is finite.

Proposition 5.4. Let $(W, \mathcal{T}, a)$ be an open, orientable, aspherical TMC. If $(W, \mathcal{T})$ has uniform isoperimetric profile and $a$ is uniformly representable, then $(W, \mathcal{T}, a)$ admits a bounded cyclic homotopy.

Proof. The construction of the cyclic homotopy $H$ is by successive extensions over the $i$-skeleta. At each step, the problem of extending $H$ from the $i$-skeleton to the $(i+1)$-skeleton can be viewed as a filling problem whose solution is provided by one of our hypotheses.

First use uniform representability to construct $H$ on the 0-skeleton. By Lemma 5.3, we have diam $H \leq C_{0}$. Then for each edge $e$ between vertices $v, v^{\prime}$, consider the combinatorial loop $\alpha_{e}$ based at $v$ defined by first following the track $H(v, \cdot)$, then running through $e$, following $H\left(v^{\prime}, \cdot\right)$ in the opposite direction and coming back through $e$. By construction, $\alpha_{e}$ is null-homotopic and has length at most $2 C_{0}+2$. Since $(W, \mathcal{T})$ has uniform isoperimetric profile, $\alpha_{e}$ can be filled by a combinatorial disc of area at most $C_{1}\left(2 C_{0}+2\right)$. This allows us to extend $H$ to a cyclic homotopy of diameter at most $C_{1}\left(2 C_{0}+2\right)$ on the 1-skeleton.

By Lemma 5.1, $(W, \mathcal{T})$ is uniformly aspherical. For each 2 -simplex $c$, the restriction of $H$ to $\partial c$ can be viewed as a combinatorial map of an annulus to $W$. This map can be extended to a combinatorial map $f: S^{2} \rightarrow W$ by adding two copies of $c$. Now by Lemma [5.3 $\operatorname{diam}(f) \leq \operatorname{Vol}_{2}(f) \leq 3 C_{1}\left(2 C_{0}+2\right)+2$. So by Lemma 5.2, the map $f$ can be filled by a map of the 3-ball of diameter at most $C_{2}\left(3 C_{1}\left(2 C_{0}+2\right)+2\right)$.

The extension of $H$ to the 3-skeleton is analogous to the last paragraph, again using uniform asphericity via Lemma 5.2 It yields a cyclic homotopy $H$ such that $\operatorname{diam} H \leq C_{2}\left(4 C_{2}\left(3 C_{1}\left(2 C_{0}+2\right)+2\right)+2\right)$.

5.3. Submanifolds of $W$ with good properties. In this subsection, let $(W, \mathcal{T}, a)$ be an open, orientable, irreducible TMC such that $a$ is uniformly representable and $(W, \mathcal{T})$ has uniform isoperimetric profile. We denote by $d$ the quasimetric coming from $\mathcal{T}$. By Proposition [5.4, there exists a bounded cyclic homotopy $H$ on $(W, \mathcal{T}, a)$.

We introduce some nice properties that a compact submanifold of $W$ may or may not have. For each property, our goal is to prove that $W$ admits an exhaustion by 
submanifolds with this property, and whenever possible, that any compact subset $K$ is contained in some compact submanifold $L$ with this property and such that the size of $L$ is bounded above by a function of the size of $K$.

\subsubsection{Regular submanifolds.}

Definition. Let $V \subset W$ be a compact, connected submanifold. We say that $V$ is regular if it is irreducible and contains a loop representing $a \in \pi_{1} W$.

One basic property of a regular submanifold is that it cannot lie in a 3-ball. This has useful consequences, e.g. every component of the complement is irreducible. We state a more general lemma.

Lemma 5.5. Let $Q$ be a connected (possibly noncompact) submanifold of $W$. Let $K_{1}, \ldots, K_{n}$ be pairwise disjoint regular submanifolds of Int $Q$. If $Q$ is irreducible, then each component of $Q-\bigcup_{i} \operatorname{Int} K_{i}$ is irreducible.

Proof. Let $X$ be a component of $Q-\bigcup_{i} \operatorname{Int} K_{i}$. Let $S$ be an embedded 2-sphere in $X$. Since $Q$ is irreducible, $S$ bounds a ball $B \subset Q$. If $B$ did not lie in $X$, it would contain some $K_{i}$, contradicting the fact that $K_{i}$ contains a loop representing $a \in \pi_{1} W$. Hence $X$ is irreducible.

Lemma 5.6. There is a function $r_{1}: \mathbf{N} \rightarrow \mathbf{N}$ such that for any compact $K \subset$ $W$, there is a regular submanifold $L \subset W$ such that $K \subset \operatorname{Int} L$ and $\operatorname{diam} L \leq$ $r_{1}(\operatorname{diam} K)$.

Proof. This is a straightforward consequence of uniform representability of $a$ and Lemma 5.1 define $r_{1}(t):=C_{2}\left(t+C_{0}+3\right)$. Let $K \subset W$ be a compact subset. Let $Y$ be the 0-neighborhood of $K$. Pick a combinatorial loop $\alpha$ representing $a$, of diameter $\leq C_{0}$, based at some vertex of $Y$. By Lemma 5.1 $Y \cup \alpha$ is contained in the interior of some aspherical submanifold $L \subset W$ of diameter at most $r_{1}(\operatorname{diam} K)$. Since $W$ is irreducible, $L$ is also irreducible, hence regular.

\subsubsection{Thick submanifolds and $S$-regular submanifolds.}

Define $C_{4}:=r_{1}(0)+\operatorname{diam} H+5$.

Definition. Let $V$ be a submanifold of $W$. We say that $V$ is thick if it is regular and contains a ball of radius $C_{4}$.

Lemma 5.7. Let $V \subset W$ be a thick submanifold. Then $V$ contains a regular submanifold whose distance to $\partial V$ is at least diam $H+1$.

Proof. Obvious from Lemma 5.6 and the definition of $C_{4}$.

Lemma 5.8. Let $K \subset W$ be a thick submanifold. Then there is a thick submanifold $L \subset W$ such that $L$ is the union of $K$ and zero or more components of $W-K$, $\operatorname{diam} L \leq \operatorname{diam} K+2 C_{4}+10$ and each compact component of $W-\operatorname{Int} L$ is thick.

Proof. Let $L$ be the union of $K$ and each compact component of $W-\operatorname{Int} K$ whose diameter is at most $C_{4}+1$. Obviously $L$ contains a ball of radius $C_{4}, \operatorname{diam} L \leq$ diam $K+2 C_{4}+10$, and each compact component of $W-\operatorname{Int} L$ contains a ball of radius $C_{4}$.

Let us prove by contradiction that $L$ is irreducible: if $L$ is reducible, then there is a ball $B \subset W$ such that $\partial B \subset L$ and $B$ is not contained in $L$. Hence some 
component $X$ of $W-\operatorname{Int} L$ lies in $B$. This $X$ has to be compact; so it contains a ball of radius $C_{4}$, hence a track of $H$. This is a contradiction.

Therefore $L$ is irreducible. Lemma 5.5 shows that each compact component of $W-$ Int $L$ is irreducible, hence a thick submanifold.

Definition. Let $V \subset W$ be a compact submanifold. We say that $V$ is $S$-regular if it is regular and admits a compatible Seifert fibration. An S-regular submanifold is taut if it is a solid torus or incompressible in $W$.

Lemma 5.9. Let $V \subset W$ be an $S$-regular submanifold.

(i) If $V$ is not a solid torus, then the compatible Seifert fibration on $V$ is unique up to isotopy.

(ii) The union of $V$ and all components of $W-\operatorname{Int} V$ that are solid tori is a taut $S$-regular submanifold.

Proof. Proof of (i): The only Seifert fiber spaces with orientable base for which uniqueness of the Seifert fibration up to isotopy fails are $S^{1} \times D^{2}, T^{2} \times I$ and $K^{2} \tilde{\times} I$ (the twisted $I$-bundle over the Klein bottle). In the last two cases, there will still be only one compatible fibration.

Proof of (ii): The union of $V$ and all components of $W-\operatorname{Int} V$ that are solid tori is S-regular because any compatible fibration on $V$ has noncontractible fiber in $W$; so it extends to the complementary solid tori. We will complete the proof by proving the following claim:

Claim. If an S-regular submanifold $U$ is not taut, then at least one component of $W-\operatorname{Int} U$ is a solid torus.

Write $\partial U=T_{1} \cup \cdots \cup T_{n} \cup T_{n+1} \cup \cdots \cup T_{n+m}$, where $T_{1}, \ldots, T_{n}$ are compressible in $W$ and $T_{n+1}, \ldots, T_{n+m}$ are incompressible. For each $1 \leq i \leq n$, choose a compression disc $D_{i}$ for $T_{i}$. Compression surgery along $D_{i}$ gives a sphere $S_{i}$ that bounds a ball $B_{i}$. Since $T_{i}$ is a union of fibers in a compatible Seifert fibration, it cannot lie in a ball. So it bounds a solid torus $V_{i}$ obtained by gluing back the two copies of $D_{i}$ in $B_{i}$. Now, by hypothesis, $U$ is not a solid torus; so there exists $i$ such that $T_{i}$ is a component of $W-\operatorname{Int} U$.

Lemma 5.10. Let $V_{1}, \ldots, V_{n}$ be pairwise disjoint thick $S$-regular submanifolds. Let $Q$ be a taut $S$-regular submanifold such that each $V_{i}$ lies in the interior of $Q$. Define $X:=Q-\bigcup_{i}$ Int $V_{i}$. If $X$ contains a ball of radius $C_{4}$, then $X$ is a thick $S$-regular submanifold.

Proof. First note that by Lemma 5.5, $X$ is irreducible; so it is a thick submanifold. By Lemma 5.7, there exists for each $i$ a regular submanifold $K_{i} \subset V_{i}$ such that $d\left(K_{i}, \partial V_{i}\right)>\operatorname{diam} H$. Set $Y:=W-\bigcup_{i} \operatorname{Int} K_{i}$. By Lemma 5.5, $Y$ is irreducible.

Let $i: X \rightarrow W$ and $j: X \rightarrow Y$ be the inclusion maps. It is easy to see that each boundary component of $X$ is incompressible in $Y$. This implies that the map $j_{*}: \pi_{1} X \rightarrow \pi_{1} Y$ is injective.

In order to apply Theorem 4.1, we are looking for a central element $A \in \pi_{1} X$ such that $i_{*} A=a$. By hypothesis, we can choose a basepoint $x_{0} \in X$ such that the track $\alpha$ of $x_{0}$ by $H$ lies in $X$. Let $A \in \pi_{1} X$ be the homotopy class of $\alpha$. By hypothesis, $H\left(X \times S^{1}\right) \subset Y$. We deduce the following equalities between subgroups 
of $\pi_{1} Y$ :

$$
\begin{aligned}
H_{*}\left(\pi_{1}\left(X \times S^{1}\right)\right) & =H_{*}\left(\pi_{1}(X \times\{0\})\right) \cdot H_{*}\left(\pi_{1}\left(\left\{x_{0}\right\} \times S^{1}\right)\right) \\
& =H_{*}\left(\pi_{1}(X \times\{0\})\right) \\
& =j_{*} \pi_{1} X .
\end{aligned}
$$

Now $j_{*} A$ is central in $H_{*}\left(\pi_{1}\left(X \times S^{1}\right)\right) \subset \pi_{1} Y$. Since $j_{*}$ is injective, we conclude that $A$ is central in $\pi_{1} X$. Moreover, $i_{*} A=a$ since $A$ is the homotopy class of a track of $H$. By Theorem 4.1, $X$ has a compatible Seifert fibration.

Lemma 5.11. Let $V \subset W$ be a thick submanifold. If each boundary component of $V$ is a torus and each compact component of $W-\operatorname{Int} V$ is thick, then $V$ is $S$-regular.

Proof. If $V$ is a solid torus, then the regularity of $V$ implies that the central element $a$ is a power of the core of $V$. So $V$ certainly admits a compatible Seifert fibration.

If $V$ is incompressible, then the map $\pi_{1} V \rightarrow \pi_{1} W$ is injective. So Theorem 4.1 gives a compatible Seifert fibration on $V$.

In the following, assume that $V$ is neither a solid torus nor incompressible. Let $T_{1}, \ldots, T_{n}$ be the components of $\partial V$ that are compressible. We claim that each $T_{i}$ bounds a (unique) solid torus $V_{i}$ that either contains $V$ or intersects $V$ in $T_{i}$.

Indeed, let $Y_{i}$ be the component of $W-\operatorname{Int} V$ that contains $T_{i}$. Then $Y_{i}$ is either noncompact or thick; so it does not lie in a ball. Now compressibility of $T_{i}$ implies that $T_{i}$ separates $W$ into two submanifolds $W_{1}, W_{2}$, one of which contains $V$ and the other $Y_{i}$. Exactly one of $W_{1}, W_{2}$ is compact. Since they both cannot lie in a ball, the compact one is a solid torus $V_{i}$.

We say that $T_{i}$ is exterior if $V \subset V_{i}$ and interior otherwise. Note that there is at most one exterior boundary component.

If there exists $i_{0}$ such that $T_{i_{0}}$ is exterior, then apply Lemma 5.10 with $Q=V_{i_{0}}$ and the thick, S-regular submanifolds are the other $V_{i}$ 's. If all $T_{i}$ 's are interior, then apply the same lemma with $Q=V \cup \bigcup_{i} V_{i}$. This finishes the proof.

\subsubsection{Existence of thick $S$-regular submanifolds. The next goal is:}

Proposition 5.12. There is a function $r_{3}: \mathbf{N} \rightarrow \mathbf{N}$ such that for any compact $K \subset W$, there is a thick $S$-regular submanifold $V \subset W$ containing $K$ in its interior such that $\operatorname{diam} V \leq r_{3}(\operatorname{diam} K)$ and each compact component of $W-\operatorname{Int} V$ is thick.

For this we need a lemma.

Lemma 5.13. There is a function $r_{2}: \mathbf{N} \rightarrow \mathbf{N}$ such that for any compact $K \in W$, there is a thick regular submanifold $U \subset W$ containing $K$ in its interior, of diameter at most $r_{2}(\operatorname{diam} K)$, and whose boundary is a union of tori.

Proof. Let $x$ be a point of $K$. By Lemma [5.6 $K \cup B\left(x, C_{4}\right)$ is contained in the interior of some regular submanifold $K^{\prime}$ of diameter at most $r_{1}$ (diam $\left.K+2 C_{4}+3\right)$. In particular, $K^{\prime}$ is thick. Applying Lemma 5.6 two more times, we find regular submanifolds $K^{\prime \prime}, K^{\prime \prime \prime}$ of $W$ such that $K^{\prime} \subset \operatorname{Int} K^{\prime \prime}, K^{\prime \prime} \subset K^{\prime \prime \prime}, d\left(\partial K^{\prime}, \partial K^{\prime \prime}\right)>\operatorname{diam} H$, $d\left(\partial K^{\prime \prime}, \partial K^{\prime \prime \prime}\right)>\operatorname{diam} H$ and diam $K^{\prime \prime \prime}$ is bounded above by a function of diam $K$.

Let $X_{1}, \ldots, X_{p}$ be the components of $K^{\prime \prime \prime}-\operatorname{Int} K^{\prime}$. By Lemma 5.5, each $X_{i}$ is irreducible. For each $1 \leq i \leq p$, let $F_{i, 1}, \ldots, F_{i, n_{i}}$ be the components of $\partial K^{\prime \prime}$ that lie in $X_{i}$. Define a class $\omega_{i} \in H_{2}\left(X_{i}\right)$ by setting $\omega_{i}:=\sum_{j}\left[F_{i, j}\right]$. 
Claim. For each $i$, there is a finite (possibly empty) collection of pairwise disjoint embedded tori $T_{i, 1}, \ldots, T_{i, m_{i}}$, none of which is null-homologous in $X_{i}$, such that the following formula holds in $H_{2}\left(X_{i}\right)$ :

$$
\omega_{i}=\sum_{k}\left[T_{i, k}\right]
$$

Let us assume this claim for the moment. Then take the union of all tori $T_{i, k}$ for all $i$ and all $k$ and forget those that are null-homologous in $K^{\prime \prime \prime}$. The resulting collection bounds a compact submanifold $U \subset K^{\prime \prime \prime}$ containing $K^{\prime}$. It follows that $K$ lies in the interior of $U$ and that $\operatorname{diam} U$ is bounded by a function of $\operatorname{diam} K$. Let us prove that $U$ is irreducible. If $U$ is reducible, then there is a ball $B \subset K^{\prime \prime \prime}$ such that $\partial B \subset U$ and $B$ is not contained in $U$. Hence $B$ contains a component $T$ of $\partial U$. This contradicts the fact that $T$ is not null-homologous in $K^{\prime \prime \prime}$. We have proved that $U$ is irreducible; so it is a thick submanifold and the proof of Lemma 5.13 modulo the claim is complete.

Let us prove the claim. Let $i$ be fixed throughout. We assume that $\omega_{i} \neq 0$.

Lemma 5.14. For all $j$, there is a finite collection $T_{j, 1}^{\prime}, \ldots, T_{j, p_{j}}^{\prime}$ of possibly singular tori such that the following formula holds in $\mathrm{H}_{2}\left(X_{i}\right)$ :

$$
F_{i, j}=\sum_{l}\left[T_{j, l}^{\prime}\right]
$$

Proof. To simplify notation, set $F=F_{i, j}$. Recall that $F$ is a boundary component of $K^{\prime \prime}$. So the condition $d\left(\partial K^{\prime \prime}, \partial X\right)>\operatorname{diam} H$ implies that the image of $F \times S^{1}$ by $H$ is contained in $X_{i}$. Thus we may consider the restriction $H: F \times S^{1} \rightarrow X_{i}$. Choose a basepoint $x_{0}$ in $F$ and define $G=H_{*}\left(\pi_{1}\left(F \times S^{1},\left(x_{0}, 0\right)\right)\right) \subset \pi_{1}\left(X_{i}, x_{0}\right)$. Let $\alpha$ be the track of $x_{0}$. Then the homotopy class of $\alpha$ in $\pi_{1}\left(W, x_{0}\right)$ is $a$. So the homotopy class of $\alpha$ in $\pi_{1}\left(X_{i}, x_{0}\right)$ is a nonzero element, say $a^{\prime}$, which belongs to $G$ by definition. In fact, $a^{\prime}$ is central in $G$ because $\pi_{1}\left(S^{1}\right)$ is central in $\pi_{1}\left(F \times S^{1}\right)$. We have just shown that $G$ has nontrivial center.

Let $X^{\prime}$ be the covering of $X_{i}$ with $\pi_{1} X^{\prime}=G$. Since $X_{i}$ is irreducible, both $X_{i}$ and $X^{\prime}$ are aspherical. So $H_{2}\left(X^{\prime}\right)=H_{2}(G)$. Furthermore, $X^{\prime}$ is irreducible by the Equivariant Sphere Theorem [25]. Consider the inclusion $j: F \rightarrow X$. Since $G$ contains $j_{*}\left(\pi_{1} F\right), j$ factors through a map $j^{\prime}: F \rightarrow X^{\prime}$. Now the homology class $[F]$ is nonzero in $H_{2}(X)$. Taking the image of the homology class $[F] \in H_{2}(X)$ under $j_{*}^{\prime}$, we get a nontrivial element $\omega$ of $H_{2}(G)$.

Scott's Compact Core Theorem [27] provides a submanifold $X_{0}^{\prime} \subset X^{\prime}$ such that $\pi_{1} X_{0}^{\prime}=G$. Since $G$ has nontrivial center, Theorem 4.1 tells us that $X_{0}^{\prime}$ is a Seifert fiber space. Hence $G$ is the fundamental group of a compact Seifert fiber space with nonempty boundary. Therefore, Lemma 4.2 implies that the class $\omega \in H_{2}(G)$ can be represented by $\mathbf{Z}^{2}$ subgroups. So we get the desired singular tori in $X_{i}$.

Theorem 4.3 gives us a Seifert fibered (possibly disconnected) submanifold $V \subset$ $X$ that contains singular tori homotopic to the $T_{j, l}^{\prime}$ 's. Take the collection of these singular tori for all $j$ and all $l$, and call it $T_{1}^{\prime \prime}, \ldots, T_{q}^{\prime \prime}$. Let $\omega^{\prime}$ be their sum in $H_{2}(V)$. Apply Lemma 4.2 to obtain disjoint incompressible embedded tori $T_{i, 1}, \ldots, T_{i, m_{i}}$ in $V$ such that $\omega^{\prime}=\sum_{k}\left[T_{i, k}\right] \in H_{2}(V)$.

Now the sum of the homology classes of $T_{1}^{\prime \prime}, \ldots, T_{q}^{\prime \prime}$ in $H_{2}(X)$ is $\omega_{i}$ and so $\omega_{i}=$ $\sum_{k}\left[T_{i, k}\right]$. This proves the claim and finishes the proof of Lemma[5.13. 
Proof of Proposition 5.12. Define $r_{3}(t):=r_{2}(t)+2 C_{4}+10$. Let $K$ be a compact subset of $W$. By Lemma 5.13, there is a thick regular submanifold $U \subset W$ containing $K$ in its interior, of diameter at most $r_{2}(\operatorname{diam} K)$, and whose boundary is a union of tori. By Lemma 5.8, adding some complementary components of $U$ yields a thick submanifold $V$ of diameter at most $r_{3}(\operatorname{diam} K)$ such that $\partial V$ is a union of tori and each compact component of $W-\operatorname{Int} V$ is thick. By Lemma $5.11 V$ is S-regular.

\subsection{Proof of Proposition 3.1.}

Proposition 3.1. Let $(W, \mathcal{T}, a)$ be an open, orientable, uniformly irreducible, uniformly representable TMC. Then $W$ admits a compatible Seifert fibration.

Proof. By Lemma 5.12, $W$ admits an exhaustion by thick S-regular submanifolds. By capping off complementary solid tori using Lemma [5.9] we get an exhaustion $V_{0} \subset V_{1} \subset \cdots \subset V_{n} \subset \cdots$ by thick, taut, S-regular submanifolds. We may assume that for every $n$, the submanifold $X_{n}:=V_{n}-\operatorname{Int} V_{0}$ is thick.

If some $V_{n}$ contains an incompressible torus, then $V_{n}$ is incompressible. By Lemma 5.9, the compatible Seifert fibration on $V_{n}$ is unique up to isotopy. The same is true for $V_{n+1}$. Since each boundary component of $V_{n}$ is an incompressible torus, we can choose the fibration on $V_{n+1}$ so that it extends the fibration on $V_{n}$. Doing the same thing inductively for all $m \geq n$, we obtain a compatible Seifert fibration on $W$.

We are left with the hard case where each $V_{n}$ is a solid torus. Since $V_{0}$ and each $X_{n}$ are thick, we can apply Lemma 5.10 to $X_{n}$ for every $n$. This gives a compatible Seifert fibration on $X_{n}$. For $n \geq 1$, the torus $\partial V_{n}$ is incompressible in $X_{n+1}$. So by the inductive argument of the previous paragraph, we can construct a compatible Seifert fibration on $W-\operatorname{Int} V_{0}$. This fibration extends to $V_{0}$, giving a compatible Seifert fibration on $W$.

\section{The M-SPlitting}

From now on, we assume that our $\operatorname{TMC}(W, \mathcal{T}, a)$ is maximal. Our goal is to find a uniform subdivision $\mathcal{T}_{2}$ of $\mathcal{T}$ such that $\left(W, \mathcal{T}_{2}, a\right)$ admits an M-splitting.

6.1. First properties of a maximal TMC. For simplicity, we use Proposition 3.1 and we fix a compatible Seifert fibration $p: W \rightarrow B$.

Definition. Let $M$ be a submanifold of $W$. A Seifert fibration $p_{M}$ on $M$ is strongly compatible if the element of $\pi_{1} W$ represented by the generic fiber of $p_{M}$ is exactly $a$.

Note that $p$ is automatically strongly compatible, because $a$ is maximal. An example of a compatible Seifert fibration that is not strongly compatible is a trivial circle fibration of a solid torus whose core is an exceptional fiber of $p$.

Lemma 6.1. Let $M$ be a connected submanifold of $W$. If some component of $\partial M$ is incompressible in $W$, then any compatible Seifert fibration on $M$ is strongly compatible.

Proof. Let $p_{M}$ be a compatible Seifert fibration on $M$ and let $T$ be an incompressible torus that is a component of $\partial M$. The Seifert fibration $p_{M}$ restricts to a fibration of $T$. Since $T$ is incompressible in $W$, we may isotop $p$ so that $T$ is a union of fibers of $p$. So we have two fibrations of $T$. Since $T$ is $\pi_{1}$-injective, the elements of $\pi_{1} T$ represented by the fibers of these two fibrations are a power of one 
another. This implies that these two fibrations are homotopic on $T$. Hence $p_{M}$ is strongly compatible.

Lemma 6.2. Let $V \subset W$ be a thick S-regular submanifold. If each compact component of $W-\operatorname{Int} V$ is thick, then $V$ admits a strongly compatible Seifert fibration. Furthermore, such a fibration is unique up to isotopy.

Proof. Recall that in a Seifert fiber space, any root of the generic fiber is a power of some exceptional fiber. As a consequence, if $p$ has no exceptional fiber, then any compatible fibration on $V$ is strongly compatible. Hence we assume that $W$ is not $S^{1} \times \mathbf{R}^{2}$, which implies that $W$ admits an exhaustion by incompressible S-regular submanifolds.

If $\partial V$ has a component that is incompressible in $W$, then we can conclude by Lemma 6.1 So we assume that it is not the case. This means that there exist solid tori $V_{1}, \ldots, V_{n}$ such that for every $i, V_{i} \cap V$ is a boundary component of $V$, and $U:=V \cup \bigcup_{i} V_{i}$ is a solid torus.

Let $Q$ be an incompressible S-regular submanifold such that $U \subset \operatorname{Int} Q$. By Lemma [5.10, $X:=Q-\bigcup_{i}$ Int $V_{i}$ has a compatible Seifert fibration. By Lemma 6.1 this fibration is strongly compatible. Now $V$ is incompressible in $X$; so the fibration can be isotoped so that $V$ is a union of fibers. Therefore $V$ admits a strongly compatible Seifert fibration.

If $V$ is a solid torus, then it is clear that the strongly compatible fibration is unique up to isotopy. Otherwise this fact comes from Lemma 5.9] $\mathrm{i}$ ).

Note that Lemma 6.2 fails without the thickness assumptions. For instance, taking a regular neighborhood of an exceptional fiber of $p$ with a trivial circle fibration and removing (open regular neighborhoods of) at least two fibers, one obtains an S-regular submanifold that admits no strongly compatible fibration (since there is only one Seifert fibration and it is not strongly compatible).

\subsection{The tori.}

Proposition 6.3. There exist constants $C_{5}, C_{6}, C_{7} \geq 0$, a function $r_{4}: \mathbf{N} \rightarrow$ $\mathbf{N}$, a uniform subdivision $\mathcal{T}_{1}$ of $\mathcal{T}$ and a submanifold $\Sigma$, each component $M$ of $W-$ Int $\Sigma$ being endowed with a strongly compatible fibration $p_{M}$, such that the following properties hold (in the following, all distances and lengths are taken with respect to $\left.\mathcal{T}_{1}\right)$ :

(i) Each component of $\Sigma$ is combinatorial with respect to $\mathcal{T}_{1}$, thick, S-regular, and has $d_{\mathcal{T}_{1}}$-diameter at most $C_{5}$.

(ii) Each component $M$ of $W-\operatorname{Int} \Sigma$ has $C_{6}$-quasidense boundary. Furthermore, for every compact $K \subset M$, there is an $S$-regular submanifold $L \subset$ $M$ such that $K \subset \operatorname{Int} L$, $\operatorname{diam} L \leq r_{4}(\operatorname{diam} K)$, and there is a strongly compatible Seifert fibration on $M$ isotopic to $p_{M}$ such that $L$ is saturated in this fibration.

(iii) In each boundary torus $T$ of $\Sigma$, there exists a curve $c_{T}$ that is a fiber of the relevant $p_{M}$, combinatorial with respect to $\mathcal{T}_{1}$, and has length at most $C_{7}$.

Proof. Define $C_{5}^{\prime}:=r_{3}(0), C_{6}^{\prime}:=\max \left\{C_{5}^{\prime}, 3 \operatorname{diam} H+10\right\}$, and $r_{4}^{\prime}(t):=$ $r_{3}\left(t+2 C_{6}^{\prime}+C_{5}^{\prime}+10\right)$.

Start with a subset $N \subset W$ maximal with the property that for each pair of distinct points $x, y \in N$ the balls $B\left(x, C_{6}^{\prime}\right)$ and $B\left(y, C_{6}^{\prime}\right)$ are disjoint. By maximality, 
$N$ is $\left(2 C_{6}^{\prime}+1\right)$-quasidense. For each point $x \in N$, apply Proposition 5.12 with $K=\{x\}$, getting an S-regular submanifold $V_{x}$ of diameter at most $C_{5}^{\prime}$, such that $x \in$ Int $V_{x}$. Let $\alpha_{x}$ be a combinatorial loop of length $\leq C_{0}$ based at $x$ and representing $a$.

Define $\Sigma:=\bigcup_{x \in N} V_{x}$. Since $C_{5}^{\prime} \leq C_{6}^{\prime}, V_{x} \cap V_{y}=\emptyset$ for $x \neq y$; so the components of $\Sigma$ are the $V_{x}$ 's. Since $\mathcal{T}$ has bounded geometry, there are only finitely many pairs $\left(B\left(x, C_{6}^{\prime}\right), \alpha_{x}\right)$ up to combinatorial homeomorphism. So we can assume that $\partial \Sigma$ is in general position with respect to $\mathcal{T}$ and that there are finitely many components of $\Sigma$ up to combinatorial homeomorphism.

To go on, we need a relative version of Proposition 5.12, Call a submanifold $V \subset W$ compatible with $\Sigma$ if for all $x \in N$, either $V_{x} \subset \operatorname{Int} V$ or $V_{x} \cap V=\emptyset$.

Lemma 6.4. For every compact $K \subset W$, there is a thick $S$-regular submanifold $V$ compatible with $\Sigma$ such that $\operatorname{diam} V \leq r_{4}^{\prime}(\operatorname{diam} K), K \subset \operatorname{Int} V$, and each compact component of $W-\operatorname{Int} V$ is thick.

Proof. Let $K$ be a compact subset of $W$. Define $K_{1}$ to be the union of $K$ and all components of $\Sigma$ whose distance from $K$ is at most $2 C_{6}^{\prime}$. The constants have been chosen so that for all $x \in N$, either $V_{x} \subset K_{1}$ or $d\left(V_{x}, K_{1}\right) \geq 2 C_{6}^{\prime}$. By Lemma 5.13 there is a thick submanifold $U$ containing $K_{1}$ and with boundary a union of tori, such that $\operatorname{diam} U \leq r_{2}\left(K_{1}\right)$. Observe that $U$ is contained in the submanifold $K^{\prime \prime \prime}$ of that lemma, which is obtained from $K_{1}$ by repeating three times the operation of taking the (diam $H$ )-neighborhood and adding compact complementary components that lie in balls. Now each component of $\Sigma$ is regular, so does not lie in a ball. As a result, the $2 C_{4}$-neighborhood of $U$ is disjoint from those components of $\Sigma$ that do not lie in $K_{1}$. Construct $V$ from $U$ by adding small complementary components as in the proof of Lemma 5.8, so that each compact component of $W-\operatorname{Int} V$ is thick. As in the proof of Proposition 5.12 we conclude using Lemma 5.11 that $V$ is a thick S-regular manifold; by construction, $\operatorname{diam} V \leq r_{4}^{\prime}(\operatorname{diam} K)$, and so $V$ satisfies the required properties.

Let $M$ be any component of $W-\operatorname{Int} \Sigma$. Lemma 6.4 gives an exhaustion $V_{0} \subset$ $V_{1} \subset \cdots \subset W$ such that each $V_{i}$ is a thick, taut S-regular submanifold compatible with $\Sigma$. Lemma 5.10 and Lemma6.2 ensure that for each $i, V_{i} \cap M$ admits a strongly compatible Seifert fibration. By strong compatibility, there is a global fibration $p_{M}$ on $M$. Remark that for each torus $T \subset \partial M$, the fiber of $p_{M}$ is homotopic in $T$ to the generic fiber of the unique strongly compatible fibration on $\Sigma$.

Hence for all $x, x^{\prime} \in N$, if $\left(B\left(x, C_{6}^{\prime}\right), \alpha_{x}\right)$ and $\left(B\left(x^{\prime}, C_{6}^{\prime}\right), \alpha_{x^{\prime}}\right)$ are homeomorphic (as pairs), then one can choose such a homeomorphism $\phi$ so that $V_{x^{\prime}}=\phi\left(V_{x}\right)$ and for each component $T$ of $\partial V_{x}$, letting $M$ (resp. $M^{\prime}$ ) denote the component of $W-$ Int $\Sigma$ that contains $T$ (resp. $\phi(T)$ ), the image under $\phi$ of any fiber of $p_{M}$ lying in $T$ is homotopic in $\phi(T)$ to the generic fiber of $p_{M^{\prime}}$.

This ensures the existence of a uniform subdivision $\mathcal{T}_{1}$ of $\mathcal{T}$ such that $\Sigma$ is combinatorial with respect to $\mathcal{T}_{1}$ and of a constant $C_{7} \geq 0$ and a collection of curves $c_{T}$ such that (iii) holds. Since $d_{\mathcal{T}}$ and $d_{\mathcal{T}_{1}}$ are quasi-isometric, there exist constants $C_{5}, C_{6} \geq 0$ and a function $r_{4}$ such that (i) and (ii) hold.

6.3. The annulus divide: Definitions. From now on, we fix a triangulation $\mathcal{T}_{1}$ and a submanifold $\Sigma$ satisfying the conclusion of Proposition 6.3 We choose a component $M$ of $W-\operatorname{Int} \Sigma$. We still denote by $\mathcal{T}_{1}$ the triangulation on $M$ obtained by restricting $\mathcal{T}_{1}$. Since $\mathcal{T}$ has bounded geometry and $\mathcal{T}_{1}$ is a uniform subdivision 
of $\mathcal{T}, \mathcal{T}_{1}$ has bounded geometry. We endow $\mathcal{T}_{1}^{(2)}$ with a Jaco-Rubinstein metric, so that we have a notion of PL area (see the Appendix).

By abuse of notation, we still denote by $a$ the element of $\pi_{1} M$ represented by the generic fiber of $p_{M}$ (although the map $\pi_{1} M \rightarrow \pi_{1} W$ need not be injective). We know that $M$ is irreducible. It has incompressible boundary unless it is a solid torus, but then its diameter can be at most $C_{5}+C_{6}$. Since our goal is to cut $M$ into pieces of uniformly bounded size, we will assume in the following that $M$ is not a solid torus.

By definition, a vertical annulus is a properly immersed annulus $A \subset M$ such that $\pi_{1} A \rightarrow \pi_{1} M$ is injective and has image the subgroup generated by $a$. A vertical annulus is essential if it is not boundary-parallel. We will use essential vertical annuli to cut $M$. They will be constructed as unions of fibers in suitable strongly compatible fibrations of S-regular submanifolds. (Note the importance of strong compatibility.) To ensure that our annuli are disjoint and of small size, we will take them of least PL area. For convenience, we give the following definition:

Definition. An h-minimal annulus is an embedded, essential vertical annulus of least PL area in its homotopy class.

An annulus divide $E$ is a set of vertical annuli in $M$, called edges, of least PL area in their homotopy classes. It is embedded if each edge is embedded and no two edges intersect each other. It is reduced if every edge is essential and no two edges are homotopic. The pieces of $M$ split along $E$ (or simply $E$-pieces) are the components of $M-\bigcup E$. Note that the edges of an embedded, reduced annulus divide are h-minimal annuli.

We shall prove:

Proposition 6.5. There is a (possibly empty) embedded, reduced annulus divide $E(M)$ in $M$, such that the following requirements are fulfilled (all diameters are taken with respect to $\left.\mathcal{T}_{1}\right)$ :

(i) Each $E(M)$-piece has trivial $\mathrm{H}_{2}$.

(ii) The $E(M)$-pieces have uniformly bounded diameter.

(iii) The edges of $E(M)$ have uniformly bounded weight.

Moreover, the bounds in (ii) and (iii) depend only on $W$ (not on $M$ ).

The proof of Proposition 6.5 is difficult and technical. It is given in the next subsection. The reader is advised to first jump to Subsection 6.5 to see why Proposition 6.5 implies Proposition 3.2 .

\subsection{Construction of the annulus divide.}

\subsubsection{First step.}

Proposition 6.6. There is a constant $C_{8} \geq 0$ and an embedded, reduced annulus divide $E_{0}$ such that each edge of $E_{0}$ has weight at most $C_{8}$ and each $E_{0}$-piece has trivial $\mathrm{H}_{2}$.

Proof. We begin with a definition. Let $T$ be an incompressible torus. If $\alpha$ is a (proper) arc in $M$, we say that $\alpha$ kills $T$ if the intersection number of $\alpha$ and $T$ is odd. If $A$ is an essential annulus in $M$, choose a compatible Seifert fibration on $X$ such that $A, T$ are saturated and let $\eta, \xi$ be the projections of $A, T$. We say that $A$ kills $T$ if the intersection number of $\eta$ and $\xi$ is odd. Note that if $T_{1}$ and $T_{2}$ are 
homologous, then $A$ kills $T_{1}$ iff $A$ kills $T_{2}$; so it makes sense to say that $A$ kills a homology class.

Lemma 6.7. For every incompressible torus $T$ such that $[T] \neq 0 \in H_{2}(M)$, there is a least PL area annulus among all essential vertical annuli killing $T$, and any such least $P L$ area annulus is embedded. There is a constant $C_{8}$ such that any such torus is killed by some essential vertical annulus of weight at most $C_{8}$.

Proof. Let $T$ be an incompressible torus such that $[T] \neq 0 \in H_{2}(M)$. It is easy to see (and will be proven below) that $T$ is killed by some essential vertical annulus $A^{\prime}$. Assuming this for the moment, we show that there exists a least PL area annulus killing $T$. By Lemma A.1, any annulus of weight no greater than $\operatorname{wt}\left(A^{\prime}\right)$ is contained in the $\operatorname{wt}\left(A^{\prime}\right)^{2}$-neighborhood of $T$. Thus there are only finitely many normal homotopy classes of essential annuli to choose from, and the existence follows as in Proposition A.2 For embeddedness, note that any least PL area annulus among all essential annuli killing $T$ minimizes PL area in its homotopy class, and that no killing annulus can be homotopic to the double cover of a Möbius band.

Set $C_{8}^{\prime}:=12 C_{6}+13$ and $C_{8}^{\prime \prime}:=r_{4}\left(C_{8}^{\prime}\right)$.

Let $T$ be an incompressible torus such that $[T] \neq 0 \in H_{2}(M)$. Either $T$ is nonseparating, or $T$ is separating and each component of $M$ split along $T$ meets $\partial M$. In both cases, there exists a killing $\operatorname{arc} \alpha$. Assume that $\alpha$ is combinatorial of minimal length $l$, and denote its endpoints by $x, y$. Let $z$ be a point of $\alpha \cap \mathcal{T}_{1}^{(0)}$ such that the lengths of the subarcs $[x z]$ and $[z y]$ are both at least $(l-1) / 2$. Since $\partial M$ is $C_{6}$-quasidense, there must exist $w \in \partial M \cap \mathcal{T}_{1}^{(0)}$ and a combinatorial arc $\beta$ connecting $z$ and $w$ of length at most $6\left(C_{6}+1\right)$. At least one of the two $\operatorname{arcs}[x z] \cup \beta$ and $[y z] \cup \beta$ kills $T$; so, by minimality, we get $l \leq C_{8}^{\prime}$.

In particular, $\alpha$ has diameter at most $C_{8}^{\prime}$. Applying Proposition 6.3 (ii), we find an S-regular submanifold $L \subset M$ of diameter at most $r_{4}\left(C_{8}^{\prime}\right)$, containing $\alpha$ in its interior, and such that there exists a strongly compatible Seifert fibration $p: M \rightarrow B$ such that $L=p^{-1}(p(L))$.

Set $\eta:=p(\alpha)$ and $\xi:=p(T)$. Put $\eta$ and $T$ in general position, keeping $\eta$ in $p(L)$. If $\eta$ is embedded, just take $A:=p^{-1}(\eta)$. Otherwise write $\eta=\eta_{1} \cup_{w} \eta_{2} \cup_{w} \eta_{3}$ where $w$ is a double point of $\eta$. If the cardinal of $\eta_{2} \cap \xi$ is even, then replace $\eta$ by $\eta_{1} \cup \eta_{3}$. If it is odd, replace $\eta$ by $\eta_{1} \cup \eta_{2} \cup \eta_{1}^{-1}$ (where $\eta_{1}^{-1}$ is $\eta_{1}$ with the opposite orientation). After perturbation, the resulting arc has fewer double points than $\eta$ and still meets $\xi$ in an odd number of points. A finite iteration of this process yields an embedded arc. The preimage of this arc is an essential annulus of diameter at most $C_{8}^{\prime \prime}$ killing $T$.

We must give a uniform bound on the minimal weight of such an annulus. This is the most subtle part, for we do not have a bound on the size of $T$. First put $T$ in normal position. We have just shown that there is an essential annulus $A$ of diameter at most $C_{8}^{\prime \prime}$ killing $T$. Let $T^{\prime}$ be a boundary component of $M$ containing at least one boundary component of $A$. Since $\operatorname{diam} T^{\prime} \leq C_{5}$, the bounded geometry of $\mathcal{T}_{1}$ gives a bound on the size of $N\left(T^{\prime}, C_{8}^{\prime \prime}\right)$. Therefore, there is only a finite number of possibilities for the 5-tuple $\left(N\left(T^{\prime}, C_{8}^{\prime \prime}\right), T^{\prime}, M \cap N\left(T^{\prime}, C_{8}^{\prime \prime}\right), T \cap N\left(T^{\prime}, C_{8}^{\prime \prime}\right), c_{T^{\prime}}\right)$ up to combinatorial homeomorphism. In each situation, there is an annulus $A \subset$ $M \cap N\left(T^{\prime}, C_{8}^{\prime \prime}\right)$ in normal position such that one component of $\partial A$ lies in $T^{\prime}$, is freely homotopic to $c_{T^{\prime}}$ and the number of components of $A \cap T$ that are essential 
curves is odd. Select for each situation an annulus with these properties and call $\mathcal{A}$ the collection of these annuli. Let $C_{8}$ be the maximum of their weights.

Now we put the pieces together: for all $T$, there is an annulus $A$ of diameter at most $C_{8}^{\prime \prime}$ killing $T$. Some annulus $A^{\prime} \in \mathcal{A}$ in a model situation has the required property. Then pulling $A^{\prime}$ back into $W$ gives an annulus of weight at most $C_{8}$ killing $T$. Note that this annulus must be vertical because of the condition on $c_{T^{\prime}}$ and essential because no boundary-parallel annulus can kill $T$.

Let $\mathcal{L}=\left\{\left[T_{1}\right],\left[T_{2}\right], \ldots\right\}$ be a list of all homology classes of embedded incompressible tori in $M$. The divide $E_{0}$ is constructed inductively. Let $A_{1}$ be of least PL area among all essential annuli that kill $T_{1}$. Delete from the list $\mathcal{L}$ all classes that are killed by $A_{1}$. Then take the first remaining class [ $\left.T_{n_{2}}\right]$. Choose an annulus $A_{2}$ of least PL area among all essential annuli that kill $\left[T_{n_{2}}\right]$ and so on. This construction gives a sequence $\left\{A_{n}\right\}$ of essential annuli, which we can view as a divide $E_{0}$. Each $A_{n}$ is h-minimal and has weight at most $C_{8}$.

It is easy to see that $E_{0}$ is reduced; indeed, if $A_{n}$ and $A_{m}$ are homotopic, then a homology class is killed by $A_{n}$ if and only if it is killed by $A_{m}$. So, by construction, $n=m$.

To show that $E_{0}$ is embedded, assume by contradiction that two edges $A_{n}, A_{m}$ intersect. By the Meeks-Yau trick (cf. the Appendix), we may suppose that the intersection is transverse. Write $A_{n}=A_{1}^{\prime} \cup_{c} A_{2}^{\prime}$ and $A_{m}=A_{3}^{\prime} \cup_{c} A_{4}^{\prime}$. Without loss of generality, we assume that $A_{3}^{\prime}$ has least PL area among the $A_{i}$ 's. So, in particular, $\left|A_{3}^{\prime}\right| \leq\left|A_{1}^{\prime}\right|$ and $\left|A_{3}^{\prime}\right| \leq\left|A_{2}^{\prime}\right|$. Let $\left[T_{p}\right]$ be a homology class on the list $\mathcal{L}$ such that $A_{n}$ has least PL area among annuli that kill $\left[T_{p}\right]$. We can choose the representative $T_{p}$ such that $c \cap T_{p}=\emptyset$ and $T_{p}$ intersects $A_{n}$ and $A_{m}$ transversely in essential curves. Then by counting the intersection curves of $T_{p}$ with $A_{1}^{\prime}$ and $A_{2}^{\prime}$ one sees that exactly one of $A_{3}^{\prime} \cup A_{1}^{\prime}$ and $A_{3}^{\prime} \cup A_{2}^{\prime}$ kills [ $T_{p}$ ], and we get a contradiction by rounding the corner of a suitable annulus. We have shown that $E_{0}$ is embedded.

It remains to see that each $E_{0}$-piece has trivial $H_{2}$. Assume that there is a piece $X$ with $H_{2}(X) \neq 0$. We know that $X$ is Seifert fibered. So, by Lemma 4.2, it contains an incompressible torus $T$ representing a nonzero class in $H_{2}(X)$. Now $T$ must be null-homologous in $M$, for otherwise its homology class in $H_{2}(M)$ would appear on the list $\mathcal{L}$, and hence some edge of $E_{0}$ would kill $T$. Therefore, $T$ bounds a compact submanifold $Y \subset \operatorname{Int} M$. No edge of $E_{0}$ can meet $Y$; so $Y \subset X$. This contradiction completes the proof of Proposition 6.6.

6.4.2. Second step. Let $E_{0}$ be the annulus divide constructed in Proposition 6.6 Our next task is to add annuli to cut the $E_{0}$-pieces into smaller pieces of uniformly bounded diameter.

Let $X$ be an $E_{0}$-piece. By hypothesis, $H_{2}(X)=0$, but $H_{2}(\bar{X})$ might be nonzero, because there might exist an edge $A$ of $E_{0}$ such that both sides of $A$ lie in $X$. To avoid this difficulty, we define abstractly the closed-up piece $\hat{X}$ using the following construction: replace each edge of $E_{0}$ by two parallel copies of this edge, obtaining a (nonreduced) divide $E^{\prime}$. Then the "interesting" $E^{\prime}$-pieces (i.e., those that are not bounded by two parallel edges) are in bijection with the $E_{0}$-pieces. If $X$ is an $E_{0}$-piece, we define $\hat{X}$ as the closure of the corresponding $E^{\prime}$-piece, with the combinatorial structure induced by the triangulation $\mathcal{T}_{1}$ and the edges of $E^{\prime}$. We identify $X$ with the obvious subset of $\hat{X}$ and endow $\hat{X}$ with the quasimetric induced 
by the covering of $\hat{X}$ by its intersections with the 3 -simplices of $\mathcal{T}_{1}$. The intrinsic diameter of $X$ is the diameter of $X$ (or $\hat{X}$ ) in this quasimetric.

Note that $X$ and $\hat{X}$ are homotopy equivalent; in particular, $H_{2}(\hat{X})=0$.

The boundary of $\hat{X}$ naturally splits into annuli of two kinds. We call boundary annuli the components of $\Sigma \cap \partial \hat{X}$ and frontier annuli the edges of $E^{\prime}$. We write $\partial \hat{X}:=\partial_{0} \hat{X} \cup \partial_{1} \hat{X}$ where $\partial_{0} \hat{X}$ is the union of the boundary annuli and $\partial_{1}$ is the union of the frontier annuli.

A properly embedded surface or arc in $\hat{X}$ is well embedded if its boundary lies in $\partial_{0} \hat{X}$. A well-embedded arc or annulus is essential if its boundary components lie on different boundary annuli $T, T^{\prime}$ such that no frontier annulus connects $T$ and $T^{\prime}$. A cutting annulus is an essential well-embedded annulus. A cutting annulus is called c-minimal if it has least PL area among all cutting annuli connecting the same boundary annuli.

Our next goal is to prove the following proposition, which is just a reformulation of Proposition 6.5.

Proposition 6.8. There exist constants $C_{9}, C_{10} \geq 0$ and an embedded, reduced annulus divide $E$ such that each E-piece $X$ has trivial $H_{2}$ and intrinsic diameter at most $C_{10}$, and each edge has weight at most $C_{9}$.

Lemma 6.9. There is a function $r_{5}: \mathbf{N} \rightarrow \mathbf{N}$ such that if $\alpha \subset \hat{X}$ is a well-embedded arc connecting boundary annuli $T$ and $T^{\prime}$, then either $T$ and $T^{\prime}$ are connected by some frontier annulus or there exists a c-minimal cutting annulus $A \subset X$ of weight at most $r_{5}(\operatorname{size}(\alpha))$ connecting $T$ and $T^{\prime}$.

Proof. The proof follows the same outline as Lemma 6.7. The annulus is constructed as a union of fibers in a Seifert fibration of some S-regular submanifold. To ensure that the annulus lies in the good piece, it suffices to take the Seifert fibration so that the divide is saturated, which is always possible since it consists of vertical annuli.

Lemma 6.10. Let $A, A^{\prime}$ be c-minimal annuli. Suppose that $A$ connects boundary annuli $T, U$ and $A^{\prime}$ connects boundary annuli $T^{\prime}, U^{\prime}$. Then the following holds:

(i) Either $A$ and $A^{\prime}$ are disjoint or, possibly after replacing $A$ by some $A_{\epsilon}$ as in Proposition A.3, the intersection consists of just one essential curve.

(ii) If $T=T^{\prime}$, then $A$ and $A^{\prime}$ are disjoint.

Proof. Proof of (i): Assume that $A$ and $A^{\prime}$ are transverse (the general case follows using the Meeks-Yau trick). By Lemma A.3, $A \cap A^{\prime}$ is a disjoint union of essential closed curves. By way of contradiction, assume that there are at least two curves $c_{1}, c_{2} \subset A \cap A^{\prime}$. Choose them so that the annuli $A_{1} \subset A$ and $A_{1}^{\prime} \subset A^{\prime}$ bounded by $c_{1} \cup c_{2}$ meet only along $c_{1} \cup c_{2}$. By exchanging $A_{1}$ and $A_{1}^{\prime}$ and rounding the corners, we get two annuli $A^{\prime \prime}, A^{\prime \prime \prime}$ such that $A^{\prime \prime}$ connects $T$ and $U, A^{\prime \prime \prime}$ connects $T^{\prime}$ and $U^{\prime}$, and $\left|A^{\prime \prime}\right|+\left|A^{\prime \prime \prime}\right|<|A|+\left|A^{\prime}\right|$. This contradicts the c-minimality of $A$ and $A^{\prime}$.

The proof of (ii) is similar: if $A$ and $A^{\prime}$ are not disjoint, by perturbing $A$ if necessary we obtain an essential curve of intersection, perform an exchange/roundoff along this curve and obtain a contradiction.

Proof of Proposition 6.8. Here is a sketch of the proof: we start with the divide $E_{0}$ given by Proposition 6.6. We construct by transfinite induction an increasing family of divides $E_{\lambda}$. At each step, we choose a piece $X$, consider the closed-up piece 
$\hat{X}$, and split $\partial \hat{X}$ into frontier annuli and boundary annuli as explained before. To construct $E_{\lambda+1}$ from $E_{\lambda}$, we choose a frontier annulus, say $A_{0}$, paint it red, and find a collection of cutting annuli that are sufficiently far from the red annulus and split $\hat{X}$ into $p+1$ components, one of which has controlled diameter, and such that the others have only one cutting annulus in their boundary. Adding the cutting annuli to the divide yields a larger divide $E_{\lambda+1}$. The cutting annuli become frontier annuli of $E_{\lambda+1}$-pieces. So we can iterate the construction to cut those new pieces, choosing the new red annulus $A_{0}$ to be the corresponding new frontier annulus. The reason for this red annulus trick is that we want the bound on the weights of the cutting annuli not to increase at each step. This will become clear in a few paragraphs.

We define the following constants:

$$
\begin{aligned}
l & :=2 C_{6}+2 \nu\left(\mathcal{T}_{1}\right) C_{8}+3, \\
C_{9} & :=\sup \left(r_{5}\left(\nu\left(\mathcal{T}_{1}\right)^{l+1}\right), C_{8}\right), \\
s & :=C_{9}^{2}+1, \\
C_{10} & :=7 s+3 C_{5}+50 .
\end{aligned}
$$

All the divides $E_{\lambda}$ will have the following properties: $E_{\lambda}$ is an extension of every divide $E_{\mu}$ for $\mu<\lambda$; each edge of $E_{\lambda}$ has weight at most $C_{9}$ (hence diameter at most $s-1$ by Lemma A.1); for every piece $X$ of $M$ split along $E_{\lambda}$, either the intrinsic diameter of $X$ is at most $C_{10}$, or $X$ admits at most one frontier annulus that is not already an edge of $E_{0}$.

Clearly $E_{0}$ has all these properties. If $\lambda$ is a limit ordinal, we set $E_{\lambda}:=\bigcup_{\mu<\lambda} E_{\mu}$. Next is the construction of $E_{\lambda+1}$ from $E_{\lambda}$. Assume that for some ordinal $\lambda$, some piece $X$ of $E_{\lambda}$ is noncompact or has intrinsic diameter greater than $C_{10}$.

Choose a red frontier annulus $A_{0} \subset \partial_{1} \hat{X}$ according to the following instructions: if some frontier annulus of $E_{\lambda}$ is not an edge of $E_{0}$, then choose this one. Otherwise choose $A_{0}$ arbitrarily. Let $U$ be the union of $A_{0}$ and the two adjacent boundary annuli. Let $Y$ be a submanifold such that $N(U, s) \subset Y \subset N(U, s+1)$. Let $F_{0}, \ldots, F_{m}$ be the components of $\partial Y$. Since $H_{2}(\hat{X})=0$, each $F_{i}$ bounds a submanifold $Y_{i}$. We say that $F_{i}$ is exterior if $Y \subset Y_{i}$. Clearly, there is exactly one boundary component, say $F_{0}$, that is exterior. Then $F_{0}$ contains $U$. Moreover, $F_{0}$ does not fill $\partial \hat{X}$, for $F_{0}=\partial \hat{X}$ would imply $\operatorname{diam}(X) \leq 2 s+2 C_{6}+3<C_{10}$.

Claim. $F_{0}-U$ is connected.

Indeed, $U$ retracts on an essential curve $c \subset F_{0}$. Seeking a contradiction, assume that $c$ is separating. Let $F$ be one of the components of $F_{0}$ split along $c$. Thus $F$ is an orientable surface whose boundary is an essential curve in $M$. It can neither be a disc nor an annulus; so it must be compressible. After a finite number of compressions, we obtain a contradiction. This proves the claim.

Choose two points $x, y$ such that $x$ lies in one of the adjacent boundary annuli of $A_{0}$ and $y$ lies in the other one. The claim implies that there exists an arc $\gamma$ in $F_{0}-U$ connecting $x$ to $y$. We can split $\gamma$ into $\gamma_{1} \cup \xi_{1} \cup \gamma_{2} \cup \cdots \cup \xi_{p-1} \cup \gamma_{p}$ where $\gamma_{i} \subset \partial \hat{X}$ and $\xi_{i} \subset \hat{X}-N(U, s)$. Let $Z_{1}, \ldots, Z_{q}$ be the components of the closure of $\partial \hat{X} \cap N\left(F_{0}, C_{6}\right)-U$. Since $\partial \hat{X}$ is $C_{6}$-quasidense, each $\gamma_{i}$ lies in some $Z_{j(i)}$. Consider the (abstract) graph $\mathcal{G}$ whose vertices are the $Z_{j}$ 's and where there is an edge between $Z_{j}$ and $Z_{k}$ iff $N\left(Z_{j}, C_{6}\right) \cap N\left(Z_{k}, C_{6}\right)$ is not contained in $N(U, s)$ (hence nonempty). 
Lemma 6.11. There is an edge path in $\mathcal{G}$ connecting $Z_{j(i)}$ and $Z_{j(i+1)}$ for all $i$.

Proof. If $j(i)=j(i+1)$, it is obvious. Otherwise we can write

$$
\xi_{i}=\left(\xi_{i} \cap N\left(Z_{j(i)}, C_{6}\right)\right) \cup \bigcup_{k \neq j(i)}\left(\xi_{i} \cap N\left(Z_{k}, C_{6}\right)\right) .
$$

This gives $\xi_{i}$ as a union of two nonempty closed subsets. Since $\xi_{i}$ is connected, the union cannot be disjoint. This means that there is a $k \neq j(i)$ such that $\xi_{i} \cap$ $N\left(Z_{j(i)}, C_{6}\right) \cap N\left(Z_{k}, C_{6}\right) \neq \emptyset$. In particular, $Z_{j(i)}$ and $Z_{k}$ are connected by an edge in $\mathcal{G}$. If $j(i+1)=k$, we are done. Otherwise, we can repeat the same argument, putting together $j(i)$ and $k$ on one side and everything else on the other side. We get an edge between $j(i)$ or $k$ and some $k^{\prime}$. After finitely many steps, we obtain an edge path in $\mathcal{G}$ connecting $Z_{j(i)}$ and $Z_{j(i+1)}$.

Call $W$ the union of all the boundary annuli and frontier annuli that do not lie entirely within $U$ and meet $N\left(Z_{j}, C_{6}\right)$ for at least one $j$. Call $W_{1}, \ldots, W_{t}$ the components of $W$. Each $Z_{j}$ is contained in some $W_{k(j)}$.

Lemma 6.12. Let $j, j^{\prime}$ be indices such that there is an edge of $\mathcal{G}$ between $Z_{j}$ and $Z_{j^{\prime}}$. Then either $k(j)=k\left(j^{\prime}\right)$ or there are boundary annuli $T \subset W_{k(j)}$ and $T^{\prime} \subset W_{k\left(j^{\prime}\right)}$ such that either $T$ and $T^{\prime}$ are connected by a frontier annulus, or there is a cminimal cutting annulus of weight at most $C_{9}$ connecting $T$ and $T^{\prime}$.

Proof. By hypothesis, there is a point $z \in \hat{X}$ such that $d\left(z, Z_{j}\right) \leq C_{6}, d\left(z, Z_{j^{\prime}}\right) \leq C_{6}$ and $d(z, U)>s$. Assume that $k(j) \neq k\left(j^{\prime}\right)$. Then there is an arc $\alpha$ of size $\leq 2 C_{6}+1$ connecting $Z_{j}$ to $Z_{j^{\prime}}$. If one endpoint (or both endpoints) of $\alpha$ lie(s) on a frontier annulus, then we can extend $\alpha$ along the boundary, getting an arc $\alpha^{\prime}$ of diameter at most $2 C_{6}+2 \nu\left(\mathcal{T}_{1}\right) C_{8}+3=l$ connecting boundary annuli $T \subset W_{k(j)}$ and $T^{\prime} \subset W_{k\left(j^{\prime}\right)}$. Note that we have just used the fact that everything takes place away from the red annulus.

By Lemma [2.2] $\operatorname{size}\left(\alpha^{\prime}\right) \leq \nu\left(\mathcal{T}_{1}\right)^{l+1}$. So by Lemma [6.9, either $T$ and $T^{\prime}$ are connected by a frontier annulus, or there is a c-minimal cutting annulus of weight at most $C_{9}$ connecting $T$ and $T^{\prime}$.

Combining the two previous lemmas, we get the following conclusion: up to reindexing the $W_{k}$ 's, there exists a finite sequence $\left(A_{1}^{\prime}, \ldots, A_{r}^{\prime}\right)$ with the following properties:

(i) Each $A_{i}^{\prime}$ is an h-minimal annulus of weight at most $C_{9}$ (either a frontier annulus or a cutting annulus).

(ii) $W_{1}$ contains $x, W_{r}$ contains $y$ and for all $i, A_{i}^{\prime}$ connects boundary annuli $T_{i} \subset W_{i}$ and $T_{i}^{\prime} \subset W_{i+1}$.

However, some of the $A_{i}^{\prime}$ 's might intersect one another. To complete the proof, we have to make them disjoint. This is the purpose of the next lemma.

Lemma 6.13. Possibly after reindexing again the $W_{k}$ 's, there is a finite sequence $\left(A_{1}^{\prime \prime}, \ldots, A_{u}^{\prime \prime}\right)$ satisfying the same properties, and, in addition, the $A_{i}^{\prime \prime}$ 's are pairwise disjoint.

Proof. Let $\left(A_{1}^{\prime \prime}, \ldots, A_{u}^{\prime \prime}\right)$ be a finite sequence of annuli with properties (i) and (ii) above. We define the complexity of $\left(A_{1}^{\prime \prime}, \ldots, A_{u}^{\prime \prime}\right)$ as the pair $(u, \eta)$, where $u$ is the cardinal of the sequence and $\eta$ is the sum of the PL areas of the $A_{i}^{\prime \prime}$ 's. The set of all possible complexities is well ordered (by the lexicographic order). So there is a 
finite sequence $\left(A_{1}^{\prime \prime}, \ldots, A_{u}^{\prime \prime}\right)$ of minimal complexity. We are going to show that the $A_{i}^{\prime \prime \prime}$ 's are pairwise disjoint.

Assume that there are indices $i<j$ such that $A_{i}^{\prime \prime} \cap A_{j}^{\prime \prime} \neq \emptyset$. By Lemma 6.10. $A_{i}^{\prime \prime} \cap A_{j}^{\prime \prime}$ consists of a single essential curve $c$, which we may assume to be transverse.

First assume that $j=i+1$. If $\left|A\left(T_{i}^{\prime}, c\right)\right| \geq\left|A\left(T_{j}, c\right)\right|$, then replace $A_{i}^{\prime \prime}$ by an annulus obtained by rounding the corners of $A\left(T_{i}, c\right) \cup_{c} A\left(c, T_{j}\right)$. Otherwise replace $A_{j}^{\prime \prime}$ by an annulus obtained by rounding the corners of $A\left(T_{i}^{\prime}, c\right) \cup_{c} A\left(c, T_{j}^{\prime}\right)$. In each case, the resulting sequence has the same cardinal and a smaller $\eta$, since the operation of rounding corners decreases strictly the PL area. This contradicts the minimality of $\left(A_{1}^{\prime \prime}, \ldots, A_{u}^{\prime \prime}\right)$.

If $j-i>1$, then we can do the same thing and delete the $A_{k}^{\prime \prime \prime}$ 's for $i<k<j$, obtaining a sequence of smaller cardinal, which is again a contradiction.

By Lemma A.1, the $A_{i}^{\prime \prime}$ 's have diameter at most $s$. Recall that they are either cutting annuli or frontier annuli. There is at least one cutting annulus among them, because otherwise, every point of $\partial \hat{X}$ would lie in $N\left(U, 3 s+C_{5}+C_{6}+15\right)$. So every point of $\hat{X}$ would lie in $N\left(U, 3 s+C_{5}+2 C_{6}+20\right)$ and $\hat{X}$ would have diameter less than $C_{10}$, contradicting our hypothesis.

Adding the $A_{i}^{\prime \prime}$ 's to $E_{\lambda}$ yields a strictly larger embedded, reduced annulus divide $E_{\lambda+1}$ satisfying the required properties.

The result follows by transfinite induction: since there are countably many homotopy classes of essential well-embedded annuli, the construction has to stop for some countable ordinal $\lambda$. The corresponding $E_{\lambda}$ has the additional property that all $E_{\lambda}$-pieces have intrinsic diameter at most $C_{10}$.

6.5. Proof of Proposition 3.2. Let $(W, \mathcal{T}, a)$ be a TMC satisfying the hypotheses of Proposition 3.2. By Proposition 6.3, there is a uniform subdivision $\mathcal{T}_{1}$ of $\mathcal{T}$ and a combinatorial submanifold $\Sigma$ whose components are thick S-regular submanifolds of uniformly bounded diameter. By Proposition 6.5. each component $M$ of $W-\operatorname{Int} \Sigma$ admits an embedded, reduced annulus divide $E(M)$ such that edges of $E(M)$ have uniformly bounded weight and $E(M)$-pieces have trivial $H_{2}$ and uniformly bounded diameters, the bounds depending on $W$ but not on $M$.

Let $\mathcal{M}^{\prime}$ be the collection consisting of all boundary components of $\Sigma$ and all edges of all divides $E(M)$. This is a collection of tori and annuli satisfying conditions (i), (ii) and (iii) of the definition of an M-splitting.

One first problem is that the annuli of $\mathcal{M}^{\prime}$ are not combinatorial with respect to $\mathcal{T}_{1}$. So we have to find a uniform subdivision making $\mathcal{M}^{\prime}$ combinatorial. To do this, it suffices to see that there are only finitely many patterns for the intersection of $\mathcal{M}^{\prime}$ and a simplex of $\mathcal{T}_{1}$. Indeed, all annuli are normal. Let $\sigma$ be a simplex of $\mathcal{T}_{2}$. The uniform bound on the weights of the annuli of $\mathcal{M}^{\prime}$ gives a bound on the number of sheets of a given annulus that can meet $\sigma$. By Lemma A.1 there is also a uniform bound on the diameters of the annuli. Since each divide $E(M)$ is reduced (i.e., two edges are never parallel), the bound on the diameters of the annuli gives a bound on the number of different annuli that can meet $\sigma$ (as in Kneser-Haken finiteness). Thus the desired uniform subdivision exists.

The collection $\mathcal{M}^{\prime}$ almost works, the only remaining problem being that components of $\Sigma$ may have nontrivial $H_{2}$. However, there are only finitely many of them up to combinatorial homeomorphism. So it suffices to add vertical annuli where 
needed and again subdivide the triangulation to obtain a uniform subdivision $\mathcal{T}_{2}$ of $\mathcal{T}$ and an M-splitting for $\left(W, \mathcal{T}_{2}, a\right)$.

\section{Proof of Proposition 3.3}

The main results of this section are Proposition 7.1 and Proposition 7.3 below, which together imply Proposition 3.3

We need some definitions and notation. If $R$ is a surface and $\mathcal{D}$ is a (locally finite) cell decomposition of $R$, we will denote by $d_{\mathcal{D}}$ the canonical quasimetric associated to the covering of $R$ by cells of $\mathcal{D}$. We say that $\mathcal{D}$ has bounded geometry if this covering has bounded geometry. This means that there are uniform upper bounds for the degree of a vertex and the combinatorial length of a 2-cell (see below). If $\mathcal{M}$ is an M-splitting of a $\operatorname{TMC}\left(W, \mathcal{T}_{2}, a\right)$, then we denote by $d_{\mathcal{M}}$ the canonical quasimetric associated to the covering of $W$ by closures of complementary components.

Here are two preliminary remarks. Let $\left(W, \mathcal{T}_{2}, a\right)$ be an open, orientable, irreducible, maximal TMC and let $\mathcal{M}$ be an M-splitting of $\left(W, \mathcal{T}_{2}, a\right)$.

Remarks. (i) Each torus of $\mathcal{M}$ meets at least one annulus. (Otherwise some complementary component would have nontrivial $H_{2}$.)

(ii) The identity map between $\left(W, d_{\mathcal{T}_{2}}\right)$ and $\left(W, d_{\mathcal{M}}\right)$ is a quasi-isometry (because the relevant compact covers are equivalent).

Proposition 7.1. Let $\left(W, \mathcal{T}_{2}, a\right)$ be an open, orientable, irreducible TMC. Assume that $\left(W, \mathcal{T}_{2}, a\right)$ admits an $M$-splitting. Then there is a compatible Seifert fibration on $W$ with base $B$ and projection map $p: W \rightarrow B$ and a bounded geometry cell decomposition $\mathcal{D}$ on $B$ such that $p$ is a quasi-isometry between $\left(W, d_{\mathcal{T}_{2}}\right)$ and $\left(B, d_{\mathcal{D}}\right)$.

Proof. Let $\mathcal{M}$ be an M-splitting. We are going to construct a compatible Seifert fibration of $\left(W, \mathcal{T}_{2}, a\right)$ that respects $\mathcal{M}$, i.e., such that the tori and annuli of $\mathcal{M}$ are saturated.

Start with a trivial circle fibration of each annulus. Condition (iii) of the definition of an M-splitting and the first preliminary remark ensure that we can extend this to a circle fibration of all tori. It remains to construct the fibration on the complement.

For convenience, thicken each annulus of $\mathcal{M}$ and extend the fibration on the thickening. Then each closure $\bar{V}$ of a complementary component $V$ has trivial $H_{2}$. Thus $\partial V$ is a single torus. By hypothesis, $V$ is aspherical, but since $W$ is irreducible, $V$ is in fact irreducible. If $V$ is a solid torus, then the fibration on $\partial V$ extends to a Seifert fibration on $V$. Otherwise $V$ is incompressible, and Theorem 4.1 gives a compatible Seifert fibration on $V$. Since $V$ is incompressible and $a$ is maximal, this Seifert fibration is automatically strongly compatible (cf. Lemma 6.1); so we can choose it so that it extends the fibration of $\partial V$.

Let $B$ be the base surface of the Seifert fibration that we have just constructed and let $p: W \rightarrow B$ be its canonical projection. The image of $\mathcal{M}$ is the 1-skeleton of a cell decomposition $\mathcal{D}$ of $B$. Since $\mathcal{T}_{2}$ has bounded geometry and complementary components of $\mathcal{M}$ have uniformly bounded diameter, $\mathcal{D}$ has bounded geometry.

The projection $p$ is a quasi-isometry between $\left(W, d_{\mathcal{M}}\right)$ and $\left(B, d_{\mathcal{D}}\right)$. So by the second preliminary remark, it is a quasi-isometry between $\left(W, d_{\mathcal{T}_{2}}\right)$ and $\left(B, d_{\mathcal{D}}\right)$.

The next task consists in finding a Riemannian metric on the base surface $B$ that "approximates" the quasimetric associated to the cell decomposition $\mathcal{D}$. For this, 
bounded geometry will be a crucial ingredient. We need to recall some definitions (most of which are taken from [22]).

Let $R$ be a surface and let $\mathcal{D}$ be a cell decomposition of $R$. The degree of a vertex $v$ is the number of 1 -cells containing $v$. The degree of $\mathcal{D}$ is the supremum of the degrees of its 0-cells. The combinatorial boundary length of a 2-cell is the number of 1-cells in its boundary, counted with multiplicities. An n-gon is a 2-cell of combinatorial boundary length $n$.

Notice that if $\mathcal{D}$ has bounded geometry, then it has finite degree and there is a uniform upper bound on the combinatorial boundary lengths of 2-cells.

A 3-gon is either a genuine triangle or some kind of "degenerate triangle" in which the number of vertices and/or edges is less than 3 . There are four types of degenerate 3 -gons (see [22]). A cell decomposition $\mathcal{D}$ is called a pseudo-triangulation if all 2-cells are 3-gons. It is nondegenerate if no 2-cell is degenerate. Any nondegenerate pseudo-triangulation can be endowed with a canonical path metric where the 3-gons are isometric to equilateral Euclidean triangles of edge length 1 . We call this the regular piecewise Euclidean metric on $\mathcal{D}$.

Lemma 7.2. Let $R$ be a surface and let $\mathcal{D}$ be a bounded geometry cell decomposition of $R$. There is a uniform subdivision $\mathcal{D}^{\prime}$ of $\mathcal{D}$ that is a nondegenerate pseudo-triangulation of finite degree.

Proof. First get rid of 1-gons and 2-gons in the following way: add a 0-cell in the boundary of each 1-gon (so that all 1-gons become 2-gons), then transform each 2 -gon into a 3 -gon by adding a 0 -cell in its boundary. The resulting cell decomposition is of course a uniform subdivision of $\mathcal{D}$ and all its 2-cells are $n$-gons with $3 \leq n \leq C$ for some constant $C$.

Then subdivide each $n$-gon $c$ into 3 -gons by choosing a 0 -cell $v \in \partial c$ and adding a 1-cell between $v$ and each 0 -cell in $\partial c$ different from $v$ and not adjacent to $v$. Each $n$-gon is divided into $(n-2) 3$-gons. So the bounded geometry of $\mathcal{D}$ ensures that the resulting pseudo-triangulation $\mathcal{D}^{\prime \prime}$ has finite degree and is a uniform subdivision of $\mathcal{D}$.

Now $\mathcal{D}^{\prime \prime}$ can be transformed into a nondegenerate pseudo-triangulation $\mathcal{D}^{\prime}$ by a subdivision in which any 2-cell is divided into at most six 2-cells (see 22, Section 5, proof of Theorems 3.5 and 3.6). Hence $\mathcal{D}^{\prime}$ is also a uniform subdivision of $\mathcal{D}$ and has finite degree.

Proposition 7.3. Let $R$ be a surface. Any bounded geometry cell decomposition $\mathcal{D}$ of $R$ is quasi-isometric to some complete Riemannian metric.

Proof. Lemma 7.2 shows that it is enough to prove Proposition 7.3 when $\mathcal{D}$ is a nondegenerate pseudo-triangulation of finite degree. Let $d$ be the regular piecewise Euclidean metric on $(R, \mathcal{D})$. We will show that $(R, d)$ is quasi-isometric to $\left(R, d_{\mathcal{D}}\right)$. Then Lemma 5.3 of 22$]$ gives the desired conclusion.

Let $\left(X, d_{X}\right)$ be the 1 -skeleton of $\mathcal{D}$ with the intrinsic metric. According to Lemma 5.2 of 22 , it is enough to check that $\left(X, d_{X}\right)$ is quasi-isometric to $\left(R, d_{\mathcal{D}}\right)$. For all $x, y \in X$, the inequality $d_{X}(x, y) \leq d_{\mathcal{D}}(x, y)+1$ is obvious. Conversely, any minimizing path (in the sense of Section 21) between $x$ and $y$ can be pushed into the 1-skeleton; so $d_{\mathcal{D}^{\prime}}(x, y) \leq 3 d_{X}(x, y)+3$. 


\section{The NONIRREDUCIBLE CASE}

In this section we show how to adapt our arguments to deal with the case where $W$ is not assumed to be irreducible, but only aspherical. Of course, we cannot expect to prove that $W$ itself is Seifert fibered (since the Poincaré conjecture is still open), but we shall prove that $W$ is homotopy equivalent to a Seifert fiber space.

To state our main result formally, it is convenient to introduce a definition:

Definition. Let $(W, \mathcal{T}, a)$ and $\left(W^{\prime}, \mathcal{T}^{\prime}, a^{\prime}\right)$ be two TMCs. A uniformly proper homotopy equivalence between $(W, \mathcal{T}, a)$ and $\left(W^{\prime}, \mathcal{T}^{\prime}, a^{\prime}\right)$ is a map $f: W \rightarrow W^{\prime}$ with the following properties:

(i) $f$ is a proper homotopy equivalence;

(ii) $f$ is a quasi-isometry between $\left(W, d_{\mathcal{T}}\right)$ and $\left(W^{\prime}, d_{\mathcal{T}^{\prime}}\right)$;

(iii) $f_{*}(a)=a^{\prime}$.

Theorem 8.1. Let $(W, \mathcal{T}, a)$ be an open, orientable, aspherical, maximal TMC. If a is uniformly representable and $(W, \mathcal{T})$ has uniform isoperimetric profile, then there exists an open, orientable, irreducible $T M C\left(W^{\prime}, \mathcal{T}^{\prime}, a^{\prime}\right)$ that is uniformly proper homotopy equivalent to $(W, \mathcal{T}, a)$ and admits a compatible Seifert fibration with base $B$ and projection map $p: W \rightarrow B$ such that there exists a complete Riemannian metric on $B$ with respect to which $p$ is a quasi-isometry.

The proof of Theorem 8.1 breaks up into two propositions.

Proposition 8.2. Let $(W, \mathcal{T}, a)$ be an open, orientable, aspherical, maximal TMC. If a is uniformly representable and $(W, \mathcal{T})$ has uniform isoperimetric profile, then there exists an open, orientable, aspherical, maximal TMC $\left(W_{2}, \mathcal{T}_{2}, a_{2}\right)$ that is uniformly proper homotopy equivalent to $(W, \mathcal{T}, a)$ and admits an $M$-splitting.

Proposition 8.3. Let $\left(W_{2}, \mathcal{T}_{2}, a_{2}\right)$ be an open, orientable, aspherical, maximal TMC that admits an M-splitting. Then there is an open, orientable, irreducible $T M C\left(W^{\prime}, \mathcal{T}^{\prime}, a^{\prime}\right)$ that is uniformly proper homotopy equivalent to $\left(W_{2}, \mathcal{T}_{2}, a_{2}\right)$, such that there is a compatible Seifert fibration on $W^{\prime}$ with base $B$ and projection map $p: W^{\prime} \rightarrow B$ and a complete Riemannian metric on $B$ such that $p$ is a quasi-isometry.

Let $(W, \mathcal{T}, a)$ be an open, orientable, aspherical TMC. Let $V$ be a possibly disconnected submanifold of $W$ each of whose components is compact and aspherical. We call Poincaré 0-surgery the following transformation: for each reducible component $U$ of $V$, we choose an embedded 2-sphere $S_{U} \subset U$ bounding a fake ball $B_{U} \subset U$ such that $U-B_{U}$ contains no fake ball. We subdivide $\mathcal{T}$ to make each $S_{U}$ combinatorial. Then we remove each $B_{U}$ and glue in its place a genuine 3-ball, suitably triangulated. Call $\left(W^{\prime}, \mathcal{T}^{\prime}\right)$ the resulting triangulated manifold. There is a natural homotopy equivalence $\phi: W \rightarrow W^{\prime}$. Let $a^{\prime} \in \pi_{1} W^{\prime}$ be the image of $a$ under $\phi_{*}$. Then $\left(W^{\prime}, \mathcal{T}^{\prime}, a^{\prime}\right)$ is an open, orientable, aspherical TMC called the Poincaré transform of $(W, \mathcal{T}, a)$ w.r.t. $V$. We call $\phi(V)$ the irreducible transform of $V$.

This construction is especially useful when components of $V$ are combinatorial of uniformly bounded size, because in this case, the subdivision above can be taken to be uniform, so that $\phi$ is uniformly proper. We record this fact for future reference.

Fact. With the previous notation, if there is a constant $C>0$ such that each component of $V$ is combinatorial and has size at most $C$, then there exists a Poincaré 
transform of $(W, \mathcal{T}, a)$ w.r.t. $V$ that is uniformly proper homotopy equivalent to $(W, \mathcal{T}, a)$.

It is now fairly easy to adapt the proof of Proposition 3.3 to prove Proposition 8.3 Let $\mathcal{M}$ be an M-splitting of $\left(W_{2}, \mathcal{T}_{2}, a_{2}\right)$. For each component $Y$ of $W_{2}$ split along $\mathcal{M}$, let $Z_{Y}$ be a submanifold of $Y$ obtained by removing an open collar neighborhood of $\partial Y$. Let $V$ be the union of those submanifolds $Z_{Y}$ over all components of $W_{2}-\bigcup \mathcal{M}$. After passing to a uniform subdivision, $V$ is combinatorial and its components are compact, aspherical submanifolds of uniformly bounded size. Hence some Poincaré transform $\left(W^{\prime}, \mathcal{T}^{\prime}, a^{\prime}\right)$ of $\left(W_{2}, \mathcal{T}_{2}, a_{2}\right)$ w.r.t. $V$ is uniformly proper homotopy equivalent to $\left(W_{2}, \mathcal{T}_{2}, a_{2}\right)$.

Observe that $\left(W^{\prime}, \mathcal{T}^{\prime}, a^{\prime}\right)$ has a natural M-splitting $\mathcal{M}^{\prime}$ and that, by construction, all components of $W^{\prime}-\bigcup \mathcal{M}^{\prime}$ are irreducible. Thus the first part of the proof of Proposition 3.3 shows that $\left(W^{\prime}, \mathcal{T}^{\prime}, a^{\prime}\right)$ admits a strongly compatible Seifert fibration respecting $\mathcal{M}^{\prime}$. Hence it is irreducible and the rest of the proof goes through without changes.

Proof of Proposition [8.2. We have to scan through sections 5 and [6, see where irreducibility is used, and adapt the definitions and arguments.

There is one basic principle the reader should keep in mind: when trying to establish the existence of an incompressible surface $F$ with some specified properties in a submanifold $V \subset W$, one can do as follows: first perform Poincaré 0-surgery in $V$, getting a manifold $W^{\prime}$ homotopy equivalent to $W$ and an irreducible submanifold $V^{\prime} \subset W^{\prime}$ such that $V$ is homeomorphic to the connected sum of $V^{\prime}$ and a homotopy sphere. Then find a surface $F^{\prime}$ in $V^{\prime}$ with the desired properties using the irreducibility of $V^{\prime}$. Finally, isotope $F^{\prime}$ off the ball coming from the 0-surgery, so that its preimage by the homotopy equivalence is a surface $F \subset V$ with the desired properties.

Let us consider Section [5] The first mention of irreducibility appears in the definition of a regular submanifold. From this point on, the word "irreducible" should everywhere be replaced by "aspherical". Thus the definition of a regular submanifold becomes:

Definition'. Let $V \subset W$ be a compact, connected submanifold. We say that $V$ is regular if it is aspherical and contains a loop representing $a \in \pi_{1} W$.

With this new definition, Lemmas 5.5 and 5.6 remain true. It suffices to replace "ball" by "homotopy ball" in their proofs.

The definition of a thick submanifold does not require any adjustment, nor do Lemmas 5.7 and 5.8 Things become more difficult when Seifert fibrations are involved. Let us call homotopy solid torus a 3-manifold homeomorphic to the connected sum of $S^{1} \times D^{2}$ with a homotopy sphere.

Definition'. A compact submanifold $V$ of $W$ is called $S$-regular if it is regular and if some irreducible transform of $V$ admits a compatible Seifert fibration. An S-regular submanifold is taut if it is incompressible in $W$ or a homotopy solid torus.

With this modified definition, Lemma 5.9(ii) remains true, and so does Lemma 5.10. For the latter, the beginning of the proof is unchanged, except that one only knows that $X$ and $Y$ are aspherical rather than irreducible. The argument used in the irreducible case proves that $\pi_{1} X$ has nontrivial center. By applying 
Theorem 4.1 in a Poincaré transform, one shows that $X$ is S-regular in the new sense.

With similar modifications, the proof of Lemma [5.11 is still valid. As far as Proposition 5.12 is concerned, irreducibility is used twice, both times in the part of its proof corresponding to Lemma 5.14. The first time, we applied Theorem 4.1 to a manifold $X_{0}^{\prime}$ in order to study a group $G$ isomorphic to $\pi_{1}\left(X_{0}^{\prime}\right)$. In the new setting, one can only show that $X_{0}^{\prime}$ is a connected sum of a Seifert fiber space with nonempty boundary and a homotopy sphere, but this difference does not concern the group $G$. The second time, we applied Theorem 4.3. To adapt the proof, one uses the principle described above: apply Theorem 4.3 in a Poincaré transform to get the tori, then remark that they already existed in the old submanifold.

One can use these results to prove a weak analogue of Proposition 3.1 by considering an exhaustion of $W$ by taut S-regular submanifolds. The outcome is that $W$ is a connected sum of a Seifert fiber space and possibly infinitely many homotopy spheres. However, this fact does not seem to be very useful in itself.

We then have to adapt the arguments of the beginning of Section6. One difficulty comes from the absence of a global Seifert fibration on $W$. Lemma 6.1 becomes:

Lemma 6.1'. Let $M$ be a compact, connected submanifold of $W$ such that some component $T$ of $\partial M$ is incompressible in $W$. Let $W^{\prime}$ be a Poincaré transform of $W$ w.r.t. $M$. Then any compatible Seifert fibration on the irreducible transform $M^{\prime} \subset W^{\prime}$ of $M$ is strongly compatible.

For the proof, we take a taut S-regular submanifold $V^{\prime} \subset W^{\prime}$ containing $M^{\prime}$. Since $V^{\prime}$ is taut and contains an incompressible torus (the image $T^{\prime}$ of $T$ ), $V^{\prime}$ is incompressible. Since $M^{\prime}$ is Seifert fibered, it is irreducible. Hence we can perform a Poincaré 0-surgery in $V^{\prime}$ - Int $M^{\prime}$ so that the image $V^{\prime \prime}$ of $V^{\prime}$ is irreducible. By maximality of $a$ and Theorem 4.1, $V^{\prime \prime}$ admits a strongly compatible Seifert fibration. Now the surgery has been done away from $T^{\prime}$. So we get a fibration on $T^{\prime}$, which we may compare to the restriction of $p_{M^{\prime}}$, and the rest of the argument is unchanged.

Lemma 6.2'. Let $V \subset W$ be a thick $S$-regular submanifold such that each compact component of $W-\operatorname{Int} V$ is thick. Let $W^{\prime}$ be a Poincaré transform of $W$ w.r.t. $V$. Then the irreducible transform $V^{\prime} \subset W^{\prime}$ of $V$ admits a strongly compatible Seifert fibration. Furthermore, such a fibration is unique up to isotopy.

In the proof of the irreducible case, the case $W \cong S^{1} \times \mathbf{R}^{2}$ was dealt with separately. Here we say that if $W$ does not have an exhaustion by incompressible S-regular submanifolds, then it has an exhaustion by homotopy solid tori that are compact cores of $W^{\prime}$. In this case, $\pi_{1} W^{\prime}$ is cyclic generated by $a$, which is enough to conclude that the compatible fibration on $V^{\prime}$ is strongly compatible. The case where $W$ does have an exhaustion by incompressible S-regular submanifolds needs no further adjustment.

Using these two lemmas and the work done before, we obtain the following weak version of Proposition 6.3.

Proposition 8.4. There exist constants $C_{5}, C_{6}, C_{7} \geq 0$, a function $r_{4}: \mathbf{N} \rightarrow$ $\mathbf{N}$, an open, orientable, aspherical, maximal TMC $\left(W_{1}, \mathcal{T}_{1}, a_{1}\right)$ that is uniformly proper homotopy equivalent to $(W, \mathcal{T}, a)$ and a submanifold $\Sigma \subset W_{1}$, each boundary component $T$ of $\Sigma$ being endowed with an embedded closed curve $c_{T} \subset T$ such that the following properties hold: 
(i) Each component of $\Sigma$ is combinatorial with respect to $\mathcal{T}_{1}$, has diameter at most $C_{5}$ and admits a strongly compatible Seifert fibration.

(ii) Each component $M$ of $W_{1}-\operatorname{Int} \Sigma$ has $C_{6}$-quasidense boundary. Furthermore, for every compact $K \subset M$, there is an $S$-regular submanifold $L \subset M$ such that $K \subset \operatorname{Int} L, \operatorname{diam} L \leq r_{4}(\operatorname{diam} K)$, and, possibly after Poincaré 0 -surgery in $L$, there is a strongly compatible Seifert fibration on $L$ such that all curves $c_{T}$ that meet $L$ are fibers.

(iii) Each curve $c_{T}$ is a fiber of the strongly compatible Seifert fibration of $\Sigma$. Furthermore, it is combinatorial and has length at most $C_{7}$.

The main difference is that we cannot expect to prove that complementary components carry Seifert fibrations, hence the clumsy statement of property (ii). The proof is similar to that of Proposition 6.3 so we just give a sketch and point out the differences: first we use the weak form of Proposition 5.12 to construct a set of S-regular submanifolds sufficiently distant from one another and such that each complementary component has quasidense boundary. We may enlarge them so that each one is thick and has only thick complementary components. Then we take the Poincaré transform with respect to the union of these submanifolds. This gives the TMC $\left(W_{1}, \mathcal{T}_{1}, a_{1}\right)$ and the Seifert fibered submanifold $\Sigma$. It is easy to check properties (i) and (iii). For property (ii), the only difference is that we do not have the fibration $p_{M}$. One would like to do the following: take a not-too-large S-regular submanifold $V \subset W_{1}$ with the following properties: it contains $K$; it is thick; each of its complementary components is thick; it contains every component of $\Sigma$ that it intersects; each component of $V-\Sigma \cap V$ is thick. Then one would like to conclude that (possibly after 0-surgery) $V$ admits a strongly compatible Seifert fibration extending that of $V \cap \Sigma$, so that one can set $L:=V \cap M$. Unfortunately it does not make sense to speak of thick submanifolds in $W_{1}$, since we do not know what constant to choose. So we really have to take $K$ back to $W$ using the homotopy equivalence, do all this in $W$, and then take the image in $W_{1}$.

Now we turn to the second part of the program, i.e., the construction of the annulus divide. Since we no longer have the fibrations $p_{M}$, we define an annulus $A \subset$ $M$ to be vertical when any essential embedded closed curve in $A$ is freely homotopic to some curve $c_{T}$ where $T$ is a boundary torus of $M$. Note that property (ii) of Proposition 8.4 implies that all curves $c_{T} \subset \partial M$ are freely homotopic in $M$ and determine a central element of $\pi_{1} M$. So we can apply the results of the Appendix.

Otherwise the only difference is that in Lemma 6.7, we do not have a Seifert fibration on the submanifold $L$ itself. Hence we apply the usual trick: do Poincaré 0 -surgery to get the Seifert fibration, construct the annulus, and pull it back to the original manifold. To obtain a uniform bound on the weights of the annuli, observe that for fixed size $(L)$, we can choose a finite number of ways of doing the Poincaré 0 -surgery, so that the resulting irreducible submanifold belongs to a finite number of combinatorial types. The rest of the argument is the same.

\section{MANifolds With COCOMPACT GROUP ACTIONS}

In order to apply the results of Section 8 we need two propositions. The first one is a version for quasimetric spaces of a standard lemma in large scale geometry.

Proposition 9.1. Let $X$ be a path-connected topological space and $\mathcal{K}$ a locally finite compact cover of $X$ of bounded geometry. Let $\Gamma$ be a finitely generated group. If $\Gamma$ 
acts properly discontinuously and cocompactly on $X$ and the action of each element preserves $\mathcal{K}$, then $\Gamma$ is quasi-isometric to $\left(X, d_{\mathcal{K}}\right)$.

Proof. Let $S$ be a finite, symmetric generating set for $\Gamma$. Throughout this proof, $l_{S}(\gamma)$ denotes the length of an element $\gamma \in \Gamma$ with respect to $S$ and $d_{S}\left(\gamma, \gamma^{\prime}\right)=$ $l_{S}\left(\gamma^{-1} \gamma^{\prime}\right)$ is the Cayley metric.

Fix a point $x_{0} \in X$. Consider the map $\phi: \Gamma \rightarrow X$ defined by $\phi(\gamma)=\gamma x_{0}$. We are going to show that $\phi$ is a quasi-isometry.

First we show that $\phi$ has quasidense image: by cocompactness, there is a compact $Y \subset X$ such that for all $x \in X$ there exists $\gamma_{x} \in \Gamma$ such that $\gamma_{x}^{-1} x \in Y$. Without loss of generality, we assume $x_{0} \in Y$. Since $\mathcal{K}$ is locally finite, $Y$ has finite diameter $D$; so $d_{\mathcal{K}}\left(x_{0}, \gamma_{x}^{-1} x\right) \leq D$ for all $x \in X$. Since the action of $\Gamma$ preserves $\mathcal{K}$, we get $d_{\mathcal{K}}\left(\gamma x_{0}, x\right)=d_{\mathcal{K}}\left(x_{0}, \gamma_{x}^{-1} x\right) \leq D$. Hence $\phi(\Gamma)$ is $D$-quasidense in $X$.

Next we pick $\gamma, \gamma^{\prime} \in \Gamma$ and find an upper bound for $d_{\mathcal{K}}\left(\gamma x_{0}, \gamma^{\prime} x_{0}\right)$ in terms of $d_{S}\left(\gamma, \gamma^{\prime}\right)$. Write $\gamma^{-1} \gamma^{\prime}=a_{1} \cdots a_{m}$ with $m=d_{S}\left(\gamma, \gamma^{\prime}\right)$. We obtain the following estimates:

$$
\begin{aligned}
d_{\mathcal{K}}\left(\gamma x_{0}, \gamma^{\prime} x_{0}\right)= & d_{\mathcal{K}}\left(x_{0}, \gamma^{-1} \gamma^{\prime} x_{0}\right) \\
\leq & d_{\mathcal{K}}\left(x_{0}, a_{1} x_{0}\right)+d_{\mathcal{K}}\left(a_{1} x_{0}, a_{1} a_{2} x_{0}\right)+\cdots \\
& \quad+d_{\mathcal{K}}\left(a_{1} \cdots a_{m-1} x_{0}, \gamma^{-1} \gamma^{\prime} x_{0}\right)+(m-1) \\
\leq & d_{\mathcal{K}}\left(x_{0}, a_{1} x_{0}\right)+d_{\mathcal{K}}\left(x_{0}, a_{2} x_{0}\right)+\cdots \\
& \quad+d_{\mathcal{K}}\left(x_{0}, a_{m} x_{0}\right)+(m-1) \\
\leq & m\left(1+\max _{a \in S} d_{\mathcal{K}}\left(x_{0}, a x_{0}\right)\right) .
\end{aligned}
$$

For the reverse estimate, we use Lemma 2.1 pick $K_{0}, \ldots, K_{l}$ with $\gamma x_{0} \in K_{0}$, $\gamma^{\prime} x_{0} \in K_{l}, l=d_{\mathcal{K}}\left(\gamma x_{0}, \gamma^{\prime} x_{0}\right)$, and $K_{i} \cap K_{i+1} \neq \emptyset$ for all $i$. Pick $y_{i} \in K_{i}$ so that $y_{0}=\gamma x_{0}, y_{l}=\gamma^{\prime} x_{0}$ and the other $y_{i}$ 's are arbitrary. By construction, each $d_{\mathcal{K}}\left(y_{i}, y_{i+1}\right)$ is 0 or 1 . Now we use the quasidensity of $\phi(\Gamma)$ : for each $i$ we choose $\gamma_{i} \in \Gamma$ such that $d_{\mathcal{K}}\left(y_{i}, \gamma_{i} x_{0}\right) \leq D$. We may take $\gamma_{0}=\gamma$ and $\gamma_{l}=\gamma^{\prime}$. We get:

$$
d_{S}\left(\gamma, \gamma^{\prime}\right) \leq d_{S}\left(\gamma, \gamma_{1}\right)+d_{S}\left(\gamma_{1}, \gamma_{2}\right)+\cdots+d_{S}\left(\gamma_{l-1}, \gamma^{\prime}\right) .
$$

For each $i$ we have

$$
d_{\mathcal{K}}\left(\gamma_{i} x_{0}, \gamma_{i+1} x_{0}\right) \leq d_{\mathcal{K}}\left(\gamma_{i} x_{0}, y_{i}\right)+d_{\mathcal{K}}\left(y_{i}, y_{i+1}\right)+d_{\mathcal{K}}\left(y_{i+1}, \gamma_{i+1} x_{0}\right)+2 \leq 2 D+3
$$

so $d_{\mathcal{K}}\left(\gamma_{i}^{-1} \gamma_{i+1} x_{0}, x_{0}\right) \leq 2 D+3$.

Now $\Gamma$ acts properly discontinuously and any ball in $\left(X, d_{\mathcal{K}}\right)$ is compact. So the set $F$ of elements $v \in \Gamma$ such that $v B\left(x_{0}, 2 D+3\right) \cap B\left(x_{0}, 2 D+3\right) \neq \emptyset$ is finite. We have shown that each $\gamma_{i}^{-1} \gamma_{i+1}$ belongs to $F$. Therefore,

$$
d_{S}\left(\gamma, \gamma^{\prime}\right) \leq l \max _{v \in F} l_{S}(v) .
$$

The second proposition says that when a TMC admits a cocompact group action, then it has all the properties needed to apply Theorem 8.1. We state it in the relevant special case.

Proposition 9.2. Let $(W, \mathcal{T}, a)$ be an open, orientable TMC. Assume that $\pi_{1} W=$ $\langle a\rangle$ and that there is a properly discontinuous, cocompact, simplicial action of a group $\Gamma$ on $(W, \mathcal{T})$. Then $W$ is aspherical, a is uniformly representable, and $(W, \mathcal{T})$ has uniform isoperimetric profile. 
Proof. Let us first show that $a$ is uniformly representable. By cocompactness, there exists a finite subcomplex $Y$ of $W$ such that for all $x \in W$ there exists $\gamma \in \Gamma$ such that $\gamma^{-1} x \in Y$. For each vertex $x$ of $Y$, we pick a combinatorial loop based at $x$ representing $a$. Let $C_{0}$ be the maximum of the lengths of these loops. Then for any $x \in \mathcal{T}^{(0)}$, one can construct a loop based at $x$ representing $a$ of length at most $C_{0}$ by taking the image under the action of an element of $\Gamma$ of some loop based at a vertex of $Y$. This shows that $a$ is uniformly representable.

Next we prove by contradiction that $\pi_{2} W$ is trivial. By the Sphere Theorem, if $\pi_{2} W \neq 0$, then there is an embedded 2-sphere $S \subset W$ which represents a nontrivial element of $\pi_{2} W$.

If $S$ is separating, call $W_{1}, W_{2}$ the components of $W$ split along $S$. For each $i$, if $W_{i}$ is noncompact, then by uniform representability, Int $W_{i}$ contains a loop representing $a \in \pi_{1} W$. Now by van Kampen's theorem, $\pi_{1} W \cong \pi_{1} W_{1} * \pi_{1} W_{2}$. Since at least one $W_{i}$, say $W_{1}$, is noncompact, we deduce that $\pi_{1} W_{2}=0$; so $W_{2}$ is a homotopy ball. This contradicts the fact that $S$ represents a nontrivial element of $\pi_{2} W$.

If $S$ is nonseparating, let $W^{\prime}$ be the result of cutting $W$ along $S$. By van Kampen's theorem, $\pi_{1} W \cong \pi_{1} W^{\prime} * \mathbf{Z}$. Now again by uniform representability, $W^{\prime}$ contains a loop representing $a \in \pi_{1} W$, which is a contradiction.

Thus we have proven that $\pi_{2} W$ is trivial. A standard argument using the Hurewicz theorem shows that $W$ is aspherical.

To see that $(W, \mathcal{T})$ has uniform isoperimetric profile, use Lemma 5.3 and cocompactness as before to reduce the problem to a finite subcomplex.

Theorem 1.2. Let $W$ be an open, orientable 3 -manifold such that $\pi_{1} W$ is infinite cyclic. Let $\Gamma$ be a finitely generated group acting smoothly, properly discontinuously and cocompactly on $W$. Then $\Gamma$ is a virtual closed surface group.

Proof. Let $a$ be a generator of $\pi_{1} W$. Construct a triangulation $\mathcal{T}$ of $W$ as follows: let $\mathcal{O}$ be the closed orbifold $W / \Gamma$. Let $\mathcal{T}^{\prime}$ be a (finite) triangulation of $\mathcal{O}$. Lift $\mathcal{T}^{\prime}$ to a $\Gamma$-equivariant triangulation of $W$. Then $\mathcal{T}$ has automatically bounded geometry (an explicit bound on the local complexity of $\mathcal{T}$ can be given in terms of the local complexity of $\mathcal{T}^{\prime}$ and the orders of the isotropy groups). Hence $(W, \mathcal{T}, a)$ is a maximal, open, orientable TMC.

By Proposition 9.2, the hypotheses of Theorem 8.1 are satisfied. Hence there is an open, orientable, irreducible TMC $\left(W^{\prime}, \mathcal{T}^{\prime}, a^{\prime}\right)$ such that $\pi_{1} W^{\prime} \cong \mathbf{Z},\left(W, d_{\mathcal{T}}\right)$ is quasi-isometric to $\left(W^{\prime}, d_{\mathcal{T}^{\prime}}\right)$ and there is a compatible Seifert fibration on $W^{\prime}$ such that $\left(W^{\prime}, d_{\mathcal{T}^{\prime}}\right)$ is quasi-isometric to some complete Riemannian metric $h$ on the base surface $B$ of this fibration.

Since $\pi_{1} W^{\prime}$ is cyclic, $B$ must be diffeomorphic to $\mathbf{R}^{2}$. By Lemma 9.1, $\Gamma$ is quasiisometric to $\left(W, d_{\mathcal{T}}\right)$, hence to $\left(W^{\prime}, d_{\mathcal{T}^{\prime}}\right)$, hence to $(B, h)$. By the characterization of groups quasi-isometric to planes [26], [22], [6], $\Gamma$ is a virtual closed surface group.

\section{Applications to ORBIFOLDS}

\subsection{Background on 3-orbifolds.}

Conventions and notation. In this paper, all 3-orbifolds are connected and orientable. All 2-orbifolds are connected and locally orientable; hence their singular points are cone points. We denote the underlying space of an orbifold $X$ by $|X|$ and its singular locus by $\Sigma_{X}$. 
For general definitions on orbifolds, see [4] or Chapter XIII of [34]. Recall that closed 2-orbifolds are divided into four classes: bad, spherical, Euclidean, and hyperbolic. A closed 2-orbifold has positive Euler characteristic iff it is bad or spherical. Some 2-orbifolds have special names, which we shall use. We recall them briefly: a teardrop is a sphere with (exactly) one cone point; a spindle is a sphere with two cone points of different orders; a football is a sphere with two cone points of the same order; a turnover is a sphere with three cone points; a pillow is a sphere with four cone points of order 2 ; a discal $n$-orbifold is an $n$-orbifold that is finitely covered by the $n$-disc.

Let $\mathcal{O}$ be a 3 -orbifold and let $F \subset \mathcal{O}$ be an orientable 2 -suborbifold. We say that $F$ is compressible if either

(i) $F$ is spherical and bounds a (discal) 3 -suborbifold isomorphic to the cone on $F$, or

(ii) $F$ has nonpositive Euler characteristic and there is a discal 2-suborbifold $D$ that intersects $F$ transversely in $\partial D$ and such that $\partial D$ does not bound a discal 2-suborbifold in $F$.

We say that $F$ is incompressible if it is not compressible. Note that if $F$ is bad or a turnover of nonpositive Euler characteristic, then $F$ is always incompressible. Hence $\mathcal{O}$ is irreducible (as defined in the introduction) iff it has no incompressible orientable 2-suborbifold of positive Euler characteristic.

Two 2-suborbifolds $F, F^{\prime}$ are isotopic if the inclusion maps of pairs $\left(|F|, \Sigma_{F}\right) \rightarrow$ $\left(|\mathcal{O}|, \Sigma_{\mathcal{O}}\right)$ and $\left(\left|F^{\prime}\right|, \Sigma_{F}^{\prime}\right) \rightarrow\left(|\mathcal{O}|, \Sigma_{\mathcal{O}}\right)$ are isotopic. If $F, F^{\prime}$ are isotopic, then they are isomorphic (as orbifolds) and $F$ is incompressible iff $F^{\prime}$ is incompressible. We shall need the following fact: let $F$ be any 2-suborbifold. Let $D \subset F$ be a discal suborbifold. Let $D^{\prime}$ be a 2-suborbifold of $\mathcal{O}$ such that $D^{\prime} \cap F=\partial D^{\prime}$ and $D \cup D^{\prime}$ is a compressible spherical 2-suborbifold. Then the 2-suborbifold obtained from $F$ by removing $D$ and gluing $D^{\prime}$ is isotopic to $F$.

10.2. Equivariant theorems. To deduce properties of 3-orbifolds from properties of their fundamental groups, we will use the following two "equivariant theorems".

Theorem 10.1. Let $\mathcal{O}$ be an orientable, irreducible 3-orbifold. Then any regular cover of $\mathcal{O}$ is irreducible.

Theorem 10.2. Let $\mathcal{O}$ be an orientable, irreducible 3 -orbifold. If $\mathcal{O}$ contains no incompressible turnovers, then no regular cover of $\mathcal{O}$ contains incompressible turnovers.

It should be noted that the orbifold $\mathcal{O}$ in Theorem 10.1 is not assumed to be good. So this theorem is not a consequence of the Equivariant Sphere Theorem for manifolds. To prove Theorems 10.1 and 10.2, we need an extension to orbifolds of the theory of least PL area surfaces (see the last section of the Appendix).

Below we fix notation that will be used for the proofs of both theorems.

Let $\mathcal{O}, \mathcal{O}^{\prime}$ be 3 -orbifolds and let $p: \mathcal{O}^{\prime} \rightarrow \mathcal{O}$ be a regular covering. Let $\Gamma:=$ $\pi_{1} \mathcal{O} / \pi_{1} \mathcal{O}^{\prime}$ be the covering group. Let $\Sigma:=\Sigma_{\mathcal{O}^{\prime}}$ be the singular locus of $\mathcal{O}^{\prime}$.

We fix a $\Gamma$-invariant triangulation $\mathcal{T}$ of $\mathcal{O}^{\prime}$. Such a triangulation can be obtained as follows: choose a triangulation of $|\mathcal{O}|$ such that $\Sigma_{\mathcal{O}}$ is a subcomplex of the 1skeleton, lift it to $\mathcal{O}^{\prime}$ and take the barycentric subdivision. A suborbifold $F \subset \mathcal{O}^{\prime}$ is equivariant if for all $g \in \Gamma$, either $g F=F$ or $g F \cap F=\emptyset$.

Let $\mathcal{S}$ (resp. $\mathcal{S}^{\prime}$ ) be the set of all orientable, incompressible 2-suborbifolds of $\mathcal{O}^{\prime}$ of positive Euler characteristic in general position with respect to $\mathcal{T}$ (resp. of 
general position incompressible 2 -suborbifolds that are spheres with at most three cone points). The strategy of the proof of Theorem 10.1 (resp. Theorem 10.2) is the following: assume that $\mathcal{S}$ (resp. $\mathcal{S}^{\prime}$ ) is nonempty, show that it contains an equivariant element $F$, and use the projection of $F$ to $\mathcal{O}$ to obtain a contradiction.

Proof of Theorem 10.1. Assume that $\mathcal{S} \neq \emptyset$. We would like to find a least PL area element $F$, and show that $F$ is equivariant. However, a least PL area element in $\mathcal{S}$ need not exist, because Theorem A.5 might only give a double cover. To deal with this situation, we define the completion $\hat{\mathcal{S}}$ of $\mathcal{S}$ to be the union of $\mathcal{S}$ and the set of double covers that are normally homotopic to elements of $\mathcal{S}$.

Lemma 10.3. There is a normal, least PL area element in $\hat{\mathcal{S}}$.

Proof. We need the following lemma.

Lemma 10.4. For every $F \in \mathcal{S}$ there exists a normal 2-suborbifold $F^{\prime} \in \mathcal{S}$ such that $\left\|F^{\prime}\right\| \leq\|F\|$.

Proof. In the case of essential 2-spheres in 3-manifolds, this lemma is due to H. Kneser [20]. It is straightforward to adapt Kneser's proof to our setting. Define the complexity $c(F)$ of a general position 2-suborbifold $F \subset \mathcal{O}^{\prime}$ by setting $c(F):=(\operatorname{sing}(F), \operatorname{wt}(F), \mathrm{n}(F))$ ordered lexicographically, where $\mathrm{n}(F)$ is the sum over all 2-simplices $\sigma$ of $\mathcal{T}$ of the number of components of $\sigma \cap|F|$.

If $F \in \mathcal{S}$ is not normal, then we may apply one of Kneser's moves [14, 20]. It is easy to check that $\mathcal{S}$ is stable under these moves. (See also the proof of Theorem 12 of [10.) The complexity $c(F)$ decreases with each move. So the process ends with a normal 2-suborbifold $F^{\prime} \in \mathcal{S}$. Since the PL area also decreases, we get $\left\|F^{\prime}\right\| \leq\|F\|$.

Let us turn to the proof of Lemma 10.3. Let $\hat{\mathcal{S}}_{\mathcal{N}}$ be the subset of $\hat{\mathcal{S}}$ consisting of the normal maps. Note that $\hat{\mathcal{S}}_{\mathcal{N}}$ has the following stability property: if $f$ is an embedding or double cover normally homotopic to some element of $\hat{\mathcal{S}}_{\mathcal{N}}$, then $f \in \hat{\mathcal{S}}_{\mathcal{N}}$. Lemma 10.4 implies that $\hat{\mathcal{S}}_{\mathcal{N}}$ is not empty, and that any least PL area map in $\hat{\mathcal{S}}_{\mathcal{N}}$ will automatically be of least PL area in $\hat{\mathcal{S}}$. Next we prove the existence of a least PL area map in $\hat{\mathcal{S}}_{\mathcal{N}}$.

Since (sing, wt) takes its values in $\mathbf{N}^{2}$, it has a minimum $(s, \omega)$ over $\hat{\mathcal{S}}_{\mathcal{N}}$. Let $\mathcal{F} \subset \hat{\mathcal{S}}_{\mathcal{N}}$ be the subset consisting of the maps $f \in \hat{\mathcal{S}}_{\mathcal{N}}$ such that $(\operatorname{sing}(f), \operatorname{wt}(f))=$ $(s, \omega)$ and $f$ is the least PL area map in $\mathcal{N}(f)$. By Theorem A.4 Theorem A.5 and the stability property of $\hat{\mathcal{S}}_{\mathcal{N}}, \mathcal{F}$ is nonempty and any least PL area map in $\mathcal{F}$ will be of least PL area in $\hat{\mathcal{S}}_{\mathcal{N}}$. Now all elements of $\mathcal{F}$ have the same total weight $\omega$. So the existence of a least PL area map $f_{0} \in \mathcal{F}$ follows from Lemma A.1 and our choice of the regular Jaco-Rubinstein metric.

Our next goal is to show that $\mathcal{S}$ has an equivariant element. Let $f_{0}$ be a normal, least PL map in $\hat{\mathcal{S}}$ whose existence is guaranteed by Lemma 10.3. Since $\mathcal{T}$ is $\Gamma$ invariant and the Jaco-Rubinstein metric is regular, each translate of $f_{0}$ by the covering group is also of least PL area in $\hat{\mathcal{S}}$.

First assume that $f_{0}$ is an embedding and call $F_{0}$ its image. We are going to prove by contradiction that $F_{0}$ is equivariant. Since the action of the covering group $\Gamma$ is properly discontinuous, there are at most finitely many elements $g_{1}, \ldots, g_{p} \in \Gamma$ such that $g_{i} F_{0} \cap F_{0} \neq \emptyset$. Thus by the Meeks-Yau trick we may further assume that 
for each $i, F_{0}$ intersects $g_{i} F_{0}$ transversely in finitely many simple closed curves that avoid $\Sigma$. Each intersection curve separates $F_{0}$ (resp. $g_{i} F_{0}$ ) into two discs with at most three singular points. There are finitely many such discs. Let $D$ be of least PL area among them. Note that $D$ is innermost.

If $D \subset F_{0}$, set $F_{1}:=F_{0}$ and $F_{2}:=g_{i} F_{0}$, and otherwise set $F_{1}:=g_{i} F_{0}$ and $F_{2}:=F_{0}$, so that $D \subset F_{1}$ anyway. Let $D_{1}, D_{2}$ be the 2 -suborbifolds such that $D_{1} \cup D_{2}=F_{2}$ and $\partial D_{1}=\partial D_{2}=\partial D$. Let $F_{i}^{\prime}$ be a 2 -suborbifold obtained by rounding the corner of $D \cup D_{i}$ for $i=1,2$. Since $D$ is innermost, $F_{1}^{\prime}$ and $F_{2}^{\prime}$ are embedded. Since $D$ has smaller PL area than the other disc in $F_{1}$ with the same boundary, $D$ has at most one singular point.

Both $F_{1}^{\prime}$ and $F_{2}^{\prime}$ have smaller PL area than $F_{2}$, because by hypothesis $\|D\| \leq$ $\left\|D_{i}\right\|$ for $i=1,2$. In particular, both are spheres with at most three singular points. Since $F_{2}$ has least PL area in $\mathcal{S}$, we deduce that $F_{1}^{\prime}$ and $F_{2}^{\prime}$ do not belong to $\mathcal{S}$.

If $\operatorname{sing}(D)=0$, then

$$
\operatorname{sing}\left(F_{1}^{\prime}\right)+\operatorname{sing}\left(F_{2}^{\prime}\right)=\operatorname{sing}\left(D_{1}\right)+\operatorname{sing}\left(D_{2}\right)=\operatorname{sing}\left(F_{2}\right) \leq 3 .
$$

Assume without loss of generality that $\operatorname{sing}\left(F_{1}^{\prime}\right) \leq \operatorname{sing}\left(F_{2}^{\prime}\right)$. Then $\operatorname{sing}\left(F_{1}^{\prime}\right)$ equals 0 or 1 . If it is 0 , then $F_{1}^{\prime}$ is a compressible nonsingular sphere. Since $F_{2}$ is incompressible, $F_{2}^{\prime}$ must also be incompressible and isomorphic to $F_{2}$; so $F_{2}^{\prime}$ belongs to $\mathcal{S}$. If it is 1 , then $F_{1}^{\prime}$ is a teardrop; so it belongs to $\mathcal{S}$. In either case there is a contradiction.

If $\operatorname{sing}(D)=1$, then the constraints $\operatorname{sing}\left(D_{1}\right)+\operatorname{sing}\left(D_{2}\right) \leq 3$ and $\operatorname{sing}\left(D_{i}\right) \geq$ $\operatorname{sing}(D)$ imply that $\operatorname{sing}\left(D_{1}\right)=1$ or $\operatorname{sing}\left(D_{2}\right)=1$ (or both). There are again two cases to consider: if $\operatorname{sing}\left(F_{2}\right)=2$, then $\operatorname{sing}\left(D_{1}\right)=\operatorname{sing}\left(D_{2}\right)=1$. At least one of the $D_{i}$ 's (say $D_{1}$ ) is isomorphic to $D$. Hence $F_{1}^{\prime}$ is a football. Since $F_{1}^{\prime} \notin \mathcal{S}$, it is compressible. But then $F_{2}^{\prime}$ is incompressible and isomorphic to $F_{2}$; so $F_{2}^{\prime}$ belongs to $\mathcal{S}$, giving again a contradiction. If $\operatorname{sing}\left(F_{2}\right)=3$, then for instance $\operatorname{sing}\left(D_{1}\right)=1$ and $\operatorname{sing}\left(D_{2}\right)=2$. Then $\operatorname{sing}\left(F_{1}^{\prime}\right)=2$. If $F_{1}^{\prime}$ were a spindle, it would belong to $\mathcal{S}$. So it must be a compressible football and we conclude as before.

If $f_{0}$ is a double cover, then we can perturb it to an embedding $f_{0, \epsilon}$. The MeeksYau trick shows that if $\epsilon$ is small enough, then the image of $f_{0, \epsilon}$ is an equivariant 2-suborbifold.

At this point, we have proven that $\mathcal{S}$ contains an equivariant 2-suborbifold $F^{\prime} \subset$ $\mathcal{O}^{\prime}$. Projecting down $F^{\prime}$ to $\mathcal{O}$, we get a 2-suborbifold $F \subset \mathcal{O}$ of positive Euler characteristic.

If $F$ is orientable, then it is compressible because $\mathcal{O}$ is irreducible. Any discal 3 -suborbifold of $\mathcal{O}$ bounded by $F$ lifts to a discal 3-suborbifold of $\mathcal{O}^{\prime}$ bounded by $F^{\prime}$, contradicting the incompressibility of $F^{\prime}$.

If $F$ is nonorientable, then it is one-sided. Let $F_{\epsilon}$ be the boundary of a small regular neighborhood of $F$. Some lift of $F_{\epsilon}$ is a small perturbation of $F^{\prime}$, hence incompressible, and we get a contradiction as in the previous paragraph.

Remark. Conversely, if $\mathcal{O}^{\prime}$ is a covering of a 3-orbifold $\mathcal{O}$ and $\mathcal{O}^{\prime}$ is irreducible, then $\mathcal{O}$ is irreducible. This is easy to show for manifolds; for orbifolds, it uses the fact that smooth finite group actions on the 3-ball are standard [24], [21].

It follows from this remark that Theorem 10.1 is still true without the hypothesis that the covering is regular. Indeed, if $\mathcal{O}$ is irreducible and $\mathcal{O}^{\prime}$ is any covering of $\mathcal{O}$, then by Theorem 10.1, the universal covering of $\mathcal{O}$ is irreducible. So by the above remark, $\mathcal{O}^{\prime}$ is irreducible. Thus the complete result is the following: 
Corollary 10.5. Let $\mathcal{O}$ be an orientable 3-orbifold and let $\mathcal{O}^{\prime}$ be a covering of $\mathcal{O}$. Then $\mathcal{O}$ is irreducible if and only if $\mathcal{O}^{\prime}$ is irreducible.

However, this result is not needed here. So our proof of Theorem 1.3 is independent of [24] and 21].

Proof of Theorem 10.2. It is almost the same as that of Theorem 10.1, the class $\mathcal{S}$ being replaced with the class $\mathcal{S}^{\prime}$.

The completion $\hat{\mathcal{S}}^{\prime}$ of $\mathcal{S}^{\prime}$ is defined in the same way as the completion $\hat{\mathcal{S}}$ of $\mathcal{S}$. The proof of Lemma 10.3 is the same, using the general results of Appendix 10.4 The heart of the proof of Theorem 10.1 is the analysis of the two 2-suborbifolds obtained by surgery along an innermost transverse curve of intersection of two elements of $\mathcal{S}$. We used the fact that the underlying surface of every element of $\mathcal{S}$ is a sphere, together with some counting arguments involving numbers of singular points of certain 2-suborbifolds. The same analysis works for $\mathcal{S}^{\prime}$.

For the end of the proof of Theorem 10.1, we used the fact that the sign of the Euler characteristic is invariant under taking finite quotients, which allowed us to obtain an incompressible orientable 2-suborbifold of $\mathcal{O}$ from one in $\mathcal{O}^{\prime}$. The end of the proof of Theorem 10.2 is similar, using the fact that a 2-suborbifold of an orientable 3-orbifold that is a quotient of a turnover is a turnover.

In fact, the same proof can be applied to other classes of incompressible 2-suborbifolds, but it fails for instance for pillows (spheres with four cone points of order 2 ). Indeed, an incompressible pillow $F$ may have an essential curve $\gamma$ cutting $F$ into two discs $D_{1}, D_{2}$ with two cone points each, such that there is a disc $D$ with two cone points of order 2 satisfying $D \cap F=\partial D=\gamma$ and such that both $D_{1} \cup D$ and $D_{2} \cup D$ are compressible pillows.

\subsection{Proof of Theorem 1.3 .}

Lemma 10.6. Let $\mathcal{O}$ be a closed, orientable, irreducible 3-orbifold. Suppose that $|\mathcal{O}|$ contains an orientable, embedded, incompressible surface (either a 2-sphere that does not bound a ball or a $\pi_{1}$-injective surface of genus $\left.\geq 1\right)$. Then $\mathcal{O}$ contains an incompressible 2-suborbifold.

Proof. Cf. 31. First suppose that $|\mathcal{O}|$ is reducible. Let $F \subset \mathcal{O}$ be a 2-suborbifold such that $|F|$ is an incompressible 2 -sphere and $\operatorname{sing}(F)$ is minimal. Since $\mathcal{O}$ is irreducible, $F$ cannot be spherical. If $F$ is compressible, then there is a discal 2-suborbifold $D$ such that $\partial D=D \cap F$ and $\partial D$ separates $F$ into two 2-suborbifolds $D_{1}, D_{2}$ that are discs with at least two singular points each. Since $|F|$ is incompressible, at least one of the spheres $|D| \cup\left|D_{1}\right|$ and $|D| \cup\left|D_{2}\right|$ is incompressible. This contradicts the minimality of $\operatorname{sing}(F)$.

Suppose now that $|\mathcal{O}|$ is irreducible. Let $F \subset \mathcal{O}$ be a 2-suborbifold such that $|F|$ is an incompressible surface and $\operatorname{sing}(F)$ is minimal. As before, we assume that $F$ is compressible and look for a contradiction. This hypothesis implies the existence of a discal 2-suborbifold $D$ such that $\partial D=D \cap F$ and $\partial D$ separates $F$ into two 2 -suborbifolds one of which, say $D^{\prime}$, is a disc with at least two singular points. Let $F^{\prime}$ be the 2-suborbifold obtained from $F$ by replacing $D$ with $D^{\prime}$. Since $|\mathcal{O}|$ is irreducible, $\left|F^{\prime}\right|$ is isotopic to $|F|$, hence incompressible. This gives the expected contradiction. 
Proposition 10.7. Let $\mathcal{O}$ be a closed, orientable, irreducible 3-orbifold that contains no incompressible turnover. If $\pi_{1} \mathcal{O}$ is infinite and isomorphic to the fundamental group of a compact, orientable, irreducible Seifert-fibered 3-orbifold, then $\mathcal{O}$ is Seifert-fibered.

Proof. There exists a finite regular covering $\mathcal{O}^{\prime}$ of $\mathcal{O}$ such that $\pi_{1} \mathcal{O}^{\prime}$ is isomorphic to the fundamental group of a Seifert-fibered Haken manifold. It follows from Theorems 10.1 and 10.2 that $\mathcal{O}^{\prime}$ is irreducible and has no incompressible turnovers.

Next we show that $\mathcal{O}^{\prime}$ contains an incompressible 2-suborbifold. If $\left|\mathcal{O}^{\prime}\right|$ is reducible, this follows from Lemma 10.6 If $\left|\mathcal{O}^{\prime}\right|$ is irreducible, then since $\pi_{1} \mathcal{O}^{\prime}$ is torsion free, $\pi_{1}\left(\left|\mathcal{O}^{\prime}\right|\right)$ is isomorphic to $\pi_{1} \mathcal{O}$. So $\left|\mathcal{O}^{\prime}\right|$ is a Haken manifold, and we can again apply Lemma10.6.

Since $\mathcal{O}$ contains no incompressible turnover, we can apply a theorem of W. Dunbar $\left[10\right.$ to deduce that $\mathcal{O}^{\prime}$ admits a hierarchy. It follows that its universal covering is $\mathbf{R}^{3}$ (cf. [40], 31]). In particular, $\mathcal{O}^{\prime}$ is good. Since $\pi_{1} \mathcal{O}^{\prime}$ is torsion free, $\mathcal{O}^{\prime}$ is a manifold. By Theorem 3.1 of [29], $\mathcal{O}^{\prime}$ is Seifert-fibered. It then follows from the Meeks-Scott Theorem [23] that $\mathcal{O}$ is Seifert-fibered.

Proof of Theorem 1.3. Let $\mathcal{O}$ be a closed, orientable, irreducible 3 -orbifold without incompressible turnovers whose fundamental group admits an infinite cyclic normal subgroup $Z$. Let $\hat{\mathcal{O}}$ be the regular covering of $\mathcal{O}$ with $\pi_{1} \hat{\mathcal{O}}=Z$ and $\Gamma=\pi_{1} \mathcal{O} / Z$ the covering group.

First assume that $Z$ has finite index in $\pi_{1} \mathcal{O}$. Then $\hat{\mathcal{O}}$ is closed. By Theorems 10.1 and $10.2 \hat{\mathcal{O}}$ is irreducible and contains no incompressible turnover. By Proposition 10.7 $\hat{\mathcal{O}}$ is a manifold. Now there is no closed, orientable, irreducible 3-manifold with infinite cyclic fundamental group. Therefore, $Z$ has infinite index.

Thus $|\hat{\mathcal{O}}|$ is an orientable open 3-manifold on which the group $\Gamma$ acts properly discontinuously and cocompactly. By Theorem 1.2. $\Gamma$ is a virtual surface group. It follows that $\pi_{1} \mathcal{O}$ is isomorphic to the fundamental group of a closed, orientable, irreducible Seifert-fibered 3-orbifold. By Proposition [10.7, $\mathcal{O}$ is Seifert-fibered.

10.4. The Torus Theorem for orbifolds. We shall deduce the Torus Theorem 1.4 from these two propositions:

Proposition 10.8. Let $\mathcal{O}$ be a closed, orientable 3 -orbifold. If $\pi_{1} \mathcal{O}$ has a subgroup isomorphic to $\mathbf{Z}^{2}$, then one of the following holds:

(i) $\pi_{1} \mathcal{O}$ splits over a group that is finite, virtually $\mathbf{Z}$ or virtually $\mathbf{Z}^{2}$, or

(ii) $\mathcal{O}$ has a finite cover $\mathcal{O}^{\prime}$ such that $\pi_{1} \mathcal{O}^{\prime}$ has a normal infinite cyclic subgroup.

Proposition 10.9. Let $\mathcal{O}$ be a closed, orientable 3 -orbifold. If $\pi_{1} \mathcal{O}$ splits over a finitely generated subgroup $H$, then $\mathcal{O}$ contains an incompressible 2 -suborbifold. Moreover, if $\mathcal{O}$ is irreducible and does not contain any incompressible turnover, then $\mathcal{O}$ contains an incompressible 2-suborbifold $F$ such that $\pi_{1} F$ is isomorphic to a subgroup of $H$.

To prove Proposition 10.8, we need a few preliminary lemmas. Recall that if $(G, H)$ is a finitely generated group pair, the number of ends of $(G, H)$ can be defined as the number of ends of the quotient of a Cayley graph of $G$ by the action of $H$. 
Lemma 10.10. Let $\mathcal{Q}$ be a compact orientable 3-orbifold whose fundamental group is isomorphic to $\mathbf{Z}^{2}$. Then $|\mathcal{Q}|$ is homeomorphic to the connected sum of $T^{2} \times I$ and a homotopy 3 -sphere. In particular, $|\mathcal{Q}|$ has exactly two boundary components.

Proof. Since $\pi_{1} \mathcal{Q}$ is torsion free, $\pi_{1}(|\mathcal{Q}|)$ is isomorphic to $\pi_{1} \mathcal{Q}$, hence to $\mathbf{Z}^{2}$. In particular, $\pi_{1}(|\mathcal{Q}|)$ is neither cyclic nor a nontrivial free product. So any embedded 2 -sphere in $|\mathcal{Q}|$ bounds a homotopy ball. By Kneser's theorem, $|\mathcal{Q}|$ is a connected sum of a homotopy 3 -sphere and a compact, orientable, irreducible 3-manifold $M$ whose fundamental group is isomorphic to $\mathbf{Z}^{2}$. By Theorem 10.6 of [15], $M$ is homeomorphic to $T^{2} \times I$.

Lemma 10.11. Let $\mathcal{O}$ be a compact, orientable 3-orbifold. Let $H$ be a finitely generated subgroup of $\pi_{1} \mathcal{O}$. Let $\hat{\mathcal{O}}$ be the covering orbifold with $\pi_{1}(\hat{\mathcal{O}})=H$. Then $\mathrm{e}\left(\pi_{1} \mathcal{O}, H\right)=\mathrm{e}(|\hat{\mathcal{O}}|)$.

Proof. Let $\mathcal{T}$ be a triangulation of $\mathcal{O}$ and let $\mathcal{D}$ be a cell decomposition of $|\mathcal{O}|$ dual to $\mathcal{T}$. Let $\tilde{\mathcal{O}}$ be the universal cover of $\mathcal{O}$. Call $\tilde{\mathcal{D}}$ (resp. $\hat{\mathcal{D}}$ ) the preimage of $\mathcal{D}$ in $|\tilde{\mathcal{O}}|$ (resp. $|\hat{\mathcal{O}}|)$. Since $\mathcal{D}^{(1)} \cap \Sigma_{\mathcal{O}}=\emptyset, \pi_{1} \mathcal{O}$ acts freely on $\tilde{\mathcal{D}}^{(1)}$. Besides, the quotient of $\tilde{\mathcal{D}}^{(1)}$ by $H$ is $\hat{\mathcal{D}}^{(1)}$. By Lemma 1.2(ii) of [28], e $\left(\pi_{1} \mathcal{O}, H\right)=\mathrm{e}\left(\hat{\mathcal{D}}^{(1)}\right)$. Now the number of ends of a locally finite $\mathrm{CW}$-complex is equal to the number of ends of its 1-skeleton; so e $\left(\pi_{1} \mathcal{O}, H\right)=\mathrm{e}(|\hat{\mathcal{O}}|)$.

Lemma 10.12. Let $\mathcal{O}$ be a closed, orientable 3-orbifold. Let $H$ be a subgroup of $\pi_{1} \mathcal{O}$ isomorphic to $\mathbf{Z}^{2}$. Then $\mathrm{e}\left(\pi_{1} \mathcal{O}, H\right) \geq 2$.

Proof. Let $\hat{\mathcal{O}}$ be the covering orbifold with $\pi_{1}(\hat{\mathcal{O}})=H$. By Lemma 10.11, we need only show that $|\hat{\mathcal{O}}|$ has at least two ends. Lemma 10.10 implies that $\hat{\mathcal{O}}$ is open. Let $X$ be a compact core of $|\hat{\mathcal{O}}|$. Again by Lemma 10.10, $X$ is a connected sum of $T^{2} \times I$ with a homotopy sphere.

Let $T$ be a boundary component of $X$. Then the inclusion $T \rightarrow|\hat{\mathcal{O}}|$ induces an isomorphism on fundamental groups. It follows that $T$ is separating. Let $Y$ be a component of $|\hat{\mathcal{O}}|$ split along $T$. The isomorphism $\pi_{1} T \rightarrow \pi_{1}(|\hat{\mathcal{O}}|)$ factors through $\pi_{1} Y$, which implies that $\pi_{1} Y$ is itself isomorphic to $\mathbf{Z}^{2}$. By Lemma10.10 $Y$ cannot be compact. Hence $|\hat{\mathcal{O}}|$ has at least two ends.

Proof of Proposition 10.8. Let $H$ be a subgroup of $\pi_{1} \mathcal{O}$ isomorphic to $\mathbf{Z}^{2}$. By Lemma 10.12, e $\left(\pi_{1} \mathcal{O}, H\right) \geq 2$. Thus $H$ admits a subgroup $H^{\prime}$ such that we have $\mathrm{e}\left(\pi_{1} \mathcal{O}, H^{\prime}\right) \geq 2$ and for every infinite index subgroup $H^{\prime \prime}<H^{\prime}$, e $\left(\pi_{1} \mathcal{O}, H^{\prime}\right)<2$. Hence the pair $\left(\pi_{1} \mathcal{O}, H^{\prime}\right)$ satisfies the hypotheses of the Algebraic Torus Theorem [11].

The "simplified" version of this theorem implies that $\pi_{1} \mathcal{O}$ satisfies one of the three properties below. In each case, we shall prove that property (i) or (ii) of Proposition 10.8 holds.

Case 1. $\pi_{1} \mathcal{O}$ is virtually abelian of rank 2 or 3 . Then (ii) holds. (In fact, rank 2 is excluded by Lemma 10.10)

Case 2. $\pi_{1} \mathcal{O}$ splits over a group that is finite, virtually $\mathbf{Z}$ or virtually $\mathbf{Z}^{2}$. This means that (i) holds.

Case 3. $\pi_{1} \mathcal{O}$ has a normal subgroup $N$ that is finite or virtually infinite cyclic such that $\pi_{1} \mathcal{O} / N$ is virtually the fundamental group of a closed hyperbolic surface. Since $\pi_{1} \mathcal{O}$ contains a subgroup isomorphic to $\mathbf{Z}^{2}, N$ cannot be finite. Let $Z$ be a finite 
index infinite cyclic subgroup of $N$. Then the normalizer of $Z$ has finite index in $\pi_{1} \mathcal{O}$; so (ii) holds.

Proof of Proposition 10.9. Let $\tilde{\mathcal{O}}$ be the universal cover of $\mathcal{O}$ with covering map $p: \tilde{\mathcal{O}} \rightarrow \mathcal{O}$. To simplify notation, we write $G=\pi_{1} \mathcal{O}$ and $X=|\tilde{\mathcal{O}}|$. By hypothesis, $G$ splits over a finitely generated subgroup $H$. By Bass-Serre theory (see e.g. [9]), there exist a simplicial tree $T$ and a simplicial action without inversions of $G$ on $T$ such that edge stabilizers are conjugates of $H$ and $T / G$ has one edge and at most two vertices.

Lemma 10.13. There exist a $G$-invariant triangulation $\tilde{\mathcal{T}}$ of $\tilde{\mathcal{O}}$ and a $G$-equivariant map $f_{0}: X \rightarrow T$ that is simplicial w.r.t. $\tilde{\mathcal{T}}$.

Proof. Let $\mathcal{T}_{1}$ be a triangulation of $\mathcal{O}$ and let $\tilde{\mathcal{T}}_{1}=p^{-1}\left(\mathcal{T}_{1}\right)$ be the induced $G$ invariant triangulation of $\tilde{\mathcal{O}}$. Let $A$ be a $G$-transversal for the action of $G$ on $\tilde{\mathcal{T}}_{1}^{(0)}$, i.e., a set of lifts of the vertices of $\mathcal{T}_{1}$.

Let $x$ be a point of $A$. Since $G$ acts properly discontinuously on $X, \operatorname{Stab}_{G}(x)$ is

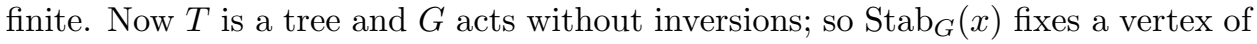
$T$. Thus we can define a map $f_{0}: A \rightarrow T$ by sending every point $x$ to a vertex of $T$ fixed by $\operatorname{Stab}_{G}(x)$.

Next we extend $f_{0}$ to $\tilde{\mathcal{T}}_{1}^{(0)}$ in the following way: for $x \in \tilde{\mathcal{T}}_{1}^{(0)}$, we choose $x_{0} \in A$ and $g \in G$ such that $x=g x_{0}$. The point $x_{0}$ is unique, but $g$ is defined only up to right multiplication by an element of $\operatorname{Stab}_{G}\left(x_{0}\right)$. We set $f_{0}(x)=g f\left(x_{0}\right)$. This is well defined because $f\left(x_{0}\right)$ is fixed by $\operatorname{Stab}_{G}\left(x_{0}\right)$.

To define $f_{0}$ on the 1 -skeleton, map every edge $\left[x_{1} x_{2}\right]$ of $\tilde{\mathcal{T}}_{1}$ to the unique (possibly degenerate) arc joining $f_{0}\left(x_{1}\right)$ and $f_{0}\left(x_{2}\right)$ by a piecewise affine map. The extensions of $f_{0}$ on the 2 -skeleton and the 3 -skeleton are similar. This construction gives a $G$-equivariant map $f_{0}: X \rightarrow T$ that is simplicial with respect to some $G$-invariant subdivision $\tilde{\mathcal{T}}$ of $\tilde{\mathcal{T}}_{1}$.

Lemma 10.14. Any continuous $G$-equivariant map $f: X \rightarrow T$ is onto.

Proof. Let $x$ be a point of $X$ and $y$ its image. Assume first that $y$ is not a vertex. Since $f$ is $G$-equivariant and $G$ acts transitively on edges, the image of the orbit of $x$ meets every edge. Now $T$ has no terminal edge, and since $f$ is continuous, $f(X)$ is connected, and so $f$ is onto.

If $y$ is a vertex, the same connectivity argument shows that $f(X)$ contains an edge; so we are reduced to the first case.

Let $f_{0}: X \rightarrow T$ be the map given by Lemma 10.13, Let $y$ be a point in $T$ that is not a vertex. Then $f_{0}^{-1}(y)$ is a 2 -submanifold of $X$, which underlies a possibly disconnected 2-suborbifold $\tilde{F}$. Since $f$ is $G$-equivariant, $\tilde{F}$ is $G$-invariant, hence $p(\tilde{F})$ is a (possibly disconnected) 2 -suborbifold $F$ of $\mathcal{O}$.

We are going to show that if some component of $F$ is compressible, then $f$ can be modified to a continuous $G$-equivariant map $f_{1}: X \rightarrow T$ such that $p\left(f_{1}^{-1}(y)\right)$ is the result of some compression surgery on $F$. After finitely many such modifications, we will get a continuous $G$-equivariant map $f_{n}: X \rightarrow T$ such that $p\left(f_{n}^{-1}(y)\right)$ is either empty or a disjoint union of incompressible 2-suborbifolds. By Lemma 10.14. $f_{n}^{-1}(y)$ cannot be empty; so $\mathcal{O}$ contains an incompressible 2 -suborbifold. 
To construct $f_{1}$, there are two cases to consider:

Case 1. Some component $S$ of $F$ is spherical and bounds a discal 3-suborbifold $Y \subset \mathcal{O}$.

Then $p^{-1}(Y)$ is a $G$-invariant disjoint union of discal 3-suborbifolds of $\tilde{\mathcal{O}}$. Let $B$ be a component of $\left|p^{-1}(Y)\right|$. Let $U$ be a $G$-equivariant regular neighborhood of $\partial B$ of the form $U=f^{-1}\left(\left[y_{-} y_{+}\right]\right)$where $y_{-}$and $y_{+}$are points of the interior of the edge containing $y$ such that $y$ lies between $y_{-}$and $y_{+}$, and $f^{-1}\left(y_{+}\right) \cap B=\emptyset$. Set $f_{1}=f$ outside $G \cdot B$ and $f(g x)=g y_{+}$for every $g \in G$ and every $x \in B$. Then $f_{1}$ is a continuous $G$-equivariant map such that $p\left(f_{1}^{-1}(y)\right)=F-S$.

Case 2. There is a discal 2-suborbifold $D$ that intersects $F$ transversely in $\partial D$ and such that $\partial D$ does not bound a discal 2-suborbifold in F.

This case is similar to Case 1. For details, see the proof of Lemma 1.1 of [39] and note that this construction can be done invariantly with respect to the action of the stabilizer of the compressing disc.

At this point, we have proven the first part of Proposition 10.9, i.e., that $\mathcal{O}$ contains an incompressible 2-suborbifold. Suppose now that $\mathcal{O}$ is irreducible and does not contain any incompressible turnover. Then by Dunbar's Theorem [10, $\mathcal{O}$ is Haken. Hence its universal cover is homeomorphic to $\mathbf{R}^{3}$ (cf. [40], [31]). In particular, $\mathcal{O}^{\prime}$ is good.

Let $F$ be a component of $p\left(f_{n}^{-1}(y)\right)$. By the Loop Theorem for good orbifolds (cf. 32]), $F$ is $\pi_{1}$-injective. Hence $\pi_{1} F$ injects into some conjugate of $H$ and the proof of Proposition 10.9 is finished.

Proof of Theorem 1.4. Let $\mathcal{O}$ be a closed, orientable, irreducible 3-orbifold containing no incompressible turnover and whose fundamental group admits a subgroup isomorphic to $\mathbf{Z}^{2}$. Apply Proposition 10.8. In case (i), we conclude from Proposition 10.9 that $\mathcal{O}$ contains an incompressible 2 -suborbifold $F$ whose fundamental group injects in a virtually $\mathbf{Z}^{2}$ subgroup. Since $\mathcal{O}$ is irreducible and closed, $F$ must be Euclidean; so we are in case (i) of Theorem 1.4 In case (ii), Theorem 1.3 implies that the finite cover $\mathcal{O}^{\prime}$ is Seifert-fibered. By passing again to a finite cover, we find a closed, orientable, irreducible Seifert manifold $M$ such that $\mathcal{O}$ is the quotient of $M$ by a finite group action. Hence by the Meeks-Scott Theorem 23, $\mathcal{O}$ is Euclidean or Seifert-fibered.

\section{Appendix. PL Minimal SURFACES AND 2-ORBIFOldS}

A.1. Normal surfaces and PL area. Let $M$ be a connected, orientable, irreducible smooth 3-manifold with a fixed smooth triangulation $\mathcal{T}$. We recall briefly some definitions from [17. Let $F$ be a compact, connected, orientable surface and let $f: F \rightarrow M$ be a proper map. We say that $f$ is a normal surface if $f(F)$ misses $\mathcal{T}^{(0)}$ and meets transversely each 3-simplex $\sigma$ of $\mathcal{T}$ in a finite collection of discs that intersect each edge of $\sigma$ in at most one point.

The weight $\operatorname{wt}(f)$ of a normal surface $f$ is the number of points of $F \cap \mathcal{T}^{(1)}$ counted with multiplicities. The normal homotopy class of a normal surface $f$ is the set of all normal surfaces $g$ that are normally homotopic to $f$, i.e., there is a (proper) homotopy $H: F \times I \rightarrow M$ such that $H_{0}=f, H_{1}=g$ and each $H_{t}$ is a normal surface.

A Jaco-Rubinstein metric on $(M, \mathcal{T})$ is a Riemannian metric on $\mathcal{T}^{(2)}-\mathcal{T}^{(0)}$ such that each 2-simplex is isometric to an ideal triangle in the hyperbolic plane $\mathbf{H}^{2}$. Such a metric is determined by the gluing maps corresponding to the edges. 
In [17, these maps were chosen more or less arbitrarily. Here we need to be more precise. Hence we fix an ideal triangle $\Delta \subset \mathbf{H}^{2}$; e.g. in the unit disc model with complex coordinates, let $\Delta$ be the triangle whose ideal vertices are the third roots of unity. We map each 2 -simplex $\sigma$ (minus its vertices) homeomorphically onto $\Delta$ using barycentric coordinates and put on $\sigma$ the induced metric, so that the gluing maps are well-defined (they belong to the isometry group of $\Delta$ ). We call this metric the regular Jaco-Rubinstein metric on $(\mathcal{O}, \mathcal{T})$.

This allows us to define the length $\lg (f)$ of a normal surface $f: F \rightarrow M$ as the total length of all the arcs in the boundaries of the discs in which $f(F)$ intersects the 3-simplices of $\mathcal{T}$. The $P L$ area of $f$ is the pair $|f|=(\operatorname{wt}(f), \lg (f)) \in \mathbf{N} \times \mathbf{R}_{+}$. We are interested in surfaces having least PL area among surfaces in a particular class with respect to the lexicographic order.

Lemma A.1. Let $F$ be a compact surface and let $f: F \rightarrow M$ be a normal map. Then size $(f(F)) \leq \operatorname{wt}(f)^{2}$.

Proof. For $i \in\{1,2,3\}$, we let $\mathcal{X}^{i}$ denote the set of the $i$-simplices of $\mathcal{T}$ that meet $f(F)$ and $n_{i}$ the cardinal of this set. Since $f$ is normal, each 3 -simplex that meets $f(F)$ has an edge that meets $f(F)$. Hence there is a map $\phi: \mathcal{X}^{3}(f) \rightarrow \mathcal{X}^{1}(f)$ such that $f(\sigma) \subset \sigma$ for all $\sigma \in \mathcal{X}^{3}(f)$. Now for all $e \in \mathcal{X}^{1}(f)$, the number of 3simplices containing $e$ is bounded by $n_{1}(f)$, hence by $\operatorname{wt}(f)$. Therefore the following inequalities hold:

$$
\operatorname{size}(f(F))=n_{3} \leq \operatorname{wt}(f) \cdot n_{1} \leq \operatorname{wt}(f)^{2} .
$$

Proposition A.2. Let $(M, \mathcal{T})$ be an orientable, aspherical triangulated 3-manifold. Let $F$ be a compact, orientable surface that is neither $S^{2}$ nor $D^{2}$. Let $f: F \rightarrow M$ be an incompressible, $\partial$-incompressible map. Then:

(i) There exists a normal map of least PL area in the homotopy class of $f$.

(ii) If $f$ is an embedding, then every least $P L$ area map homotopic to $f$ is an embedding or a double cover of a nonorientable surface.

(iii) Any two homotopically disjoint embedded surfaces of least PL area in their respective homotopy classes are disjoint or equal.

Proof. (i) is proved as in [17] except that if $M$ is noncompact, there may be infinitely many normal homotopy classes of given weight in the homotopy class of $f$. By Lemma A.1 there are only finitely many of them up to combinatorial homeomorphism. This suffices to establish the existence of a least PL area map, because we are using the regular Jaco-Rubinstein metric.

From this existence result, we deduce (ii) and (iii) as Theorems 6 and 7 of [17, i.e., as Theorems 5.1 and 6.2 of [12].

A.2. Vertical annuli in TMCs. Let $(M, \mathcal{T}, a)$ be an orientable, aspherical TMC. Assume that $M$ has nonempty incompressible boundary. A Jaco-Rubinstein metric on $\mathcal{T}^{(2)}$ is fixed once and for all. Recall that a vertical annulus is a properly immersed annulus $A \subset M$ such that $\pi_{1} A \rightarrow \pi_{1} M$ is injective and has image the subgroup generated by $a$, and that a vertical annulus is essential if it is not boundaryparallel. Hence an embedded, essential vertical annulus is incompressible and $\partial$-incompressible, which means that Proposition A.2 applies to essential vertical annuli. Recall that such an annulus is called h-minimal when it minimizes PL area in its homotopy class. 
We say that an $\operatorname{arc} \alpha$ properly embedded in $M$ is inessential if it is parallel to an arc in $\partial M$. If $A$ is an embedded essential annulus, we say that an embedded curve $c \subset A$ is inessential on $A$ if it is contractible in $A$. A (properly) embedded $\operatorname{arc} \alpha \subset A$ is essential on $A$ if it connects the two boundary components of $A$, i.e., if it is not parallel into $\partial A$. In each case, we say that something is inessential if it is not essential, and vice versa. Therefore, an embedded curve $c \subset A$ is inessential on $A$ iff it is contractible in $M$. An embedded $\operatorname{arc} \alpha \subset A$ is (in)essential on $A$ iff it is (in)essential in $M$. In particular, the property of being essential on a given essential annulus $A$ does not depend on $A$.

Two embedded essential annuli $A, A^{\prime}$ are transverse if $A \cap A^{\prime}$ is a finite, possibly empty disjoint union of curves and arcs transverse to $\mathcal{T}^{(2)}$, along which $A$ and $A^{\prime}$ intersect transversely. It can be shown that if both $A$ and $A^{\prime}$ are h-minimal, then they are transverse unless $A \cap A^{\prime}$ admits a generalized saddle point and/or an arc contained in $\mathcal{T}^{(2)}$. In that case, for small $\epsilon>0$ there is an annulus $A_{\epsilon}$ normally homotopic to $A$ such that $\lg \left(A_{\epsilon}\right) \leq \lg (A)+\epsilon$ and $A_{\epsilon}$ is transverse to $A^{\prime}$. This allows us to extend to the general case any result about PL minimal surfaces obtained in the transverse case by an exchange/roundoff argument. This is called in [17 the Meeks-Yau trick.

Assume $A, A^{\prime}$ are transverse. Each component of $A \cap A^{\prime}$ (closed curve or arc) is essential on $A$ if and only if it is essential on $A^{\prime}$; so it makes sense to speak of essential vs. inessential intersection curves (or arcs).

Lemma A.3. Let $A, A^{\prime}$ be h-minimal annuli.

(i) If $A$ and $A^{\prime}$ are transverse, then $A \cap A^{\prime}$ is empty or consists only of essential closed curves.

(ii) Otherwise, for small $\epsilon$, there exists an annulus $A_{\epsilon}$ normally homotopic to $A$ and transverse to $A^{\prime}$ such that $A_{\epsilon} \cap A^{\prime}$ is empty or consists only of essential closed curves.

Proof. We distinguish two cases.

Case 1: $A, A^{\prime}$ are transverse.

The proof is by "induction on niceness". If $A \cap A^{\prime}$ has an inessential closed component $c$, then we can choose one that is innermost on $A$, i.e., $c$ bounds a disc $D \subset A$ whose interior does not intersect $A^{\prime}$. Now $c$ also bounds a disc $D^{\prime} \subset A^{\prime}$. Thus $D \cup D^{\prime}$ is a sphere embedded in $\operatorname{Int} M$. Since $M$ is aspherical, it bounds a homotopy ball. Then a classical exchange/roundoff argument (as, e.g., in [17, Section 2.2) gives a contradiction.

Assume now that $A \cap A^{\prime}$ has no inessential closed component, but has an inessential arc component $\alpha$. Choose $\alpha$ innermost in the sense that it cobounds a disc $D \subset A$ with an $\operatorname{arc} \beta \subset \partial A$ and Int $D$ does not intersect $A^{\prime}$. Since $\alpha$ is also inessential on $A^{\prime}$, it cobounds a disc $D^{\prime} \subset A^{\prime}$ with an arc $\beta^{\prime} \subset \partial A^{\prime}$. Piecing together $\beta$ and $\beta^{\prime}$ yields a curve that bounds the disc $D \cup D^{\prime}$. Now $\partial M$ is incompressible; so $\beta \cup \beta^{\prime}$ bounds a disc $D^{\prime \prime} \subset \partial M$. We can now construct a sphere $S$ properly embedded in $M$ and isotopic to $D \cup D^{\prime} \cup D^{\prime \prime}$. Again, the asphericity of $M$ gives a contradiction using an exchange/roundoff argument.

Assume finally that $A \cap A^{\prime}$ has no inessential component, but one or more essential arc components. Then $A \cap A^{\prime}$ is a disjoint union of essential arcs. Let $F$ be a component of $\partial M$ that meets $A$ (and hence $A^{\prime}$ ). Choose boundary curves $c, c^{\prime}$ of respectively $A$ and $A^{\prime}$ such that $c$ and $c^{\prime}$ lie in $F$. Then $c$ and $c^{\prime}$ intersect 
transversely in finitely many points. Since $M$ has incompressible boundary, the map $\pi_{1} F \rightarrow \pi_{1} M$ is injective; so $c$ and $c^{\prime}$ represent the same central element in $\pi_{1} F$. Hence the number of intersection points between $c$ and $c^{\prime}$ is even. In particular, there are at least two $\operatorname{arcs}$ in $A \cap A^{\prime}$. Take two such $\operatorname{arcs} \alpha_{1}, \alpha_{2}$ with the property that there are arcs $\beta_{1}, \beta_{2}$ in $\partial A$ such that the disc $D \subset A$ bounded by $\alpha_{1} \cup \beta_{1} \cup \alpha_{2} \cup \beta_{2}$ meets $A^{\prime}$ only along $\alpha_{1} \cup \alpha_{2}$. There exist $\operatorname{arcs} \beta_{1}^{\prime}, \beta_{2}^{\prime} \subset A^{\prime}$ such that $\beta_{1} \cup \beta_{1}^{\prime}$ (resp. $\beta_{2} \cup \beta_{2}^{\prime}$ ) cobounds a disc $D_{1} \subset \partial M$ such that $D_{1} \cap D=\beta_{1}$ (resp. a disc $D_{2} \subset \partial M$ such that $D_{2} \cap D=\beta_{2}$ ) and $\alpha_{1} \cup \beta_{1}^{\prime} \cup \alpha_{2} \cup \beta_{2}^{\prime}$ bounds a disc $D^{\prime} \subset A^{\prime}$ such that $D \cup D^{\prime} \cup D_{1} \cup D_{2}$ is an embedded sphere. As before, we get a contradiction using the asphericity of $M$.

Case 2: $A, A^{\prime}$ are not transverse. We use the Meeks-Yau trick: take $\epsilon>0$ small enough so that there is an annulus $A_{\epsilon}$ normally homotopic to $A$ and transverse to $A^{\prime}$, such that any exchange/roundoff between $A_{\epsilon}$ and $A^{\prime}$ reduces length by more than $\epsilon$. In each of the previous cases, the asphericity of $M$ implies the existence of annuli $A^{\prime \prime}, A^{\prime \prime \prime}$ homotopic respectively to $A$ and $A^{\prime}$ such that

$$
\operatorname{wt}\left(A^{\prime \prime}\right)+\operatorname{wt}\left(A^{\prime \prime \prime}\right)=\operatorname{wt}(A)+\operatorname{wt}\left(A^{\prime}\right)
$$

and

$$
\lg \left(A^{\prime \prime}\right)+\lg \left(A^{\prime \prime \prime}\right)<\lg \left(A_{\epsilon}\right)+\lg \left(A^{\prime}\right)-\epsilon \leq \lg (A)+\lg \left(A^{\prime}\right) .
$$

Thus we have either $\left|A^{\prime \prime}\right|<|A|$ or $\left|A^{\prime \prime \prime}\right|<\left|A^{\prime}\right|$, which contradicts the fact that $A$ and $A^{\prime}$ are h-minimal.

A.3. PL area of orbifolds. There is some overlap between this subsection and a recent paper by Y. Takeuchi and M. Yokoyama 33.

Definition. Let $\mathcal{O}$ be a 3-orbifold. A triangulation $\mathcal{T}$ of $\mathcal{O}$ is a triangulation of the underlying manifold $|\mathcal{O}|$ such that $\Sigma_{\mathcal{O}}$ is a subcomplex of the 1-skeleton and every 3 -simplex of $\mathcal{T}$ intersects $\Sigma_{\mathcal{O}}$ in either the empty set, a vertex or an edge. The pair $(\mathcal{O}, \mathcal{T})$ is called a triangulated 3 -orbifold.

Definition. Let $\mathcal{O}$ be a 3 -orbifold with a fixed triangulation $\mathcal{T}$ and let $F$ be a closed, orientable 2-orbifold. A map $f: F \rightarrow \mathcal{O}$ is in general position (resp. normal) if the induced map $|f|:|F| \rightarrow|\mathcal{O}|$ is in general position (resp. normal) with respect to $\mathcal{T}$, and if for every $x \in F$, the local group at $f(x)$ is isomorphic to the local group at $x$. The normal homotopy class of a normal map is defined as in the manifold case.

Examples. (i) Let $f: F \rightarrow \mathcal{O}$ be a normal map. If $|f|$ is an embedding, then its image underlies a 2 -suborbifold of $\mathcal{O}$ isomorphic to $F$. Such an orbifold will be called a normal suborbifold, and the map $f$ is called an embedding. In the sequel, a normal 2-suborbifold will often be identified with its inclusion map.

(ii) Let $f: F \rightarrow \mathcal{O}$ be a normal map. If $|f|$ is a double covering onto its image, then this image underlies a one-sided nonorientable 2 -suborbifold of $\mathcal{O}$ whose orientation cover is isomorphic to $F$. We call $f$ a double cover.

Let $(\mathcal{O}, \mathcal{T})$ be a triangulated 3-orbifold. Give $\mathcal{T}^{(2)}$ the regular Jaco-Rubinstein metric on $(\mathcal{O}, \mathcal{T})$. Let $F$ be a closed, orientable 2-orbifold and $f: F \rightarrow \mathcal{O}$ a map in general position. The singular weight $\operatorname{sing}(f)$ of $f$ is the number of singular points of $F$. Its total weight wt $(f)$ (resp. length $\lg (f)$ ) is the weight (resp. length in the regular Jaco-Rubinstein metric) of the underlying map. The PL area of $f$ is the ordered triple $\|f\|:=(\operatorname{sing}(f), \operatorname{wt}(f), \lg (f)) \in \mathbf{N}^{2} \times \mathbf{R}_{+}$(with the lexicographic order). 
A few remarks are in order: the singular weight of a general position map is never greater than its total weight. Its total weight and length are the same as those of its underlying map. Normally homotopic maps have the same singular weight and total weight; so a map is of least PL area in its normal homotopy class iff it is of least length.

A linking 2-suborbifold is a normal embedded spherical 2-suborbifold $S$ that bounds a small discal 3 -suborbifold around a vertex of $\mathcal{T}$. (Equivalently, the underlying surface of $S$ is a linking sphere in the sense of [17.) A multiple of a linking 2 -suborbifold $S$ is a normal map obtained by gluing together all the triangles in some finite number of copies of $S$.

By the above remarks, the next two theorems are direct consequences of results of $[17$.

Theorem A.4 (Theorems 1 and 2 of [17]). Let $f: F \rightarrow \mathcal{O}$ be a normal map that is not a multiple of a linking 2-suborbifold. Then there is a unique least PL area map in the normal homotopy class of $f$.

Theorem A.5 (Corollary 3 of [17]). Let $f: F \rightarrow \mathcal{O}$ be a normal map that is not a multiple of a linking 2-suborbifold. If $f$ is an embedding, then the least PL area map in the normal homotopy class of $f$ is either an embedding or a double cover.

\section{ACNOWLEDGEMENTS}

Some results of this article are contained in my doctoral thesis at Université Paul Sabatier, Toulouse. I would like to thank my advisor Michel Boileau for his suggestions and guidance.

\section{REFERENCES}

1. Michel Boileau, Bernhard Leeb, and Joan Porti, On the geometry of 3-dimensional cone manifolds, Preprint math.GT/0010185, 2000.

2. Uniformization of compact orientable 3-orbifolds, Preprint math.GT/0010184, 2000.

3. Michel Boileau and Joan Porti, Geometrization of 3-orbifolds of cyclic type, Astérisque (2001), no. 272, 208, Appendix A by Michael Heusener and Joan Porti. MR 2002f:57034

4. F. Bonahon and L. C. Siebenmann, The characteristic toric splitting of irreducible compact 3-orbifolds, Math. Ann. 278 (1987), 441-479. MR 90a:57017

5. Brian H. Bowditch, A topological characterisation of hyperbolic groups, J. Amer. Math. Soc. 11 (1998), no. 3, 643-667. MR 99c:20048

6. , Planar groups and the Seifert conjecture, Preprint, November 1999.

7. Andrew Casson and Douglas Jungreis, Convergence groups and Seifert fibered 3-manifolds, Invent. Math. 118 (1994), 441-456. MR 96f:57011

8. Daryl Cooper, Craig D. Hodgson, and Steven P. Kerckhoff, Three-dimensional orbifolds and cone-manifolds, Mathematical Society of Japan Memoirs, vol. 5, Japan Publ. Trading Co., 2000. MR 2002c:57027

9. Warren Dicks and M. J. Dunwoody, Groups acting on graphs, Cambridge University Press, Cambridge, 1989. MR 91b:20001

10. William D. Dunbar, Hierarchies for 3-orbifolds, Topology Appl. 29 (1988), no. 3, 267-283. MR 89h:57008

11. M. J. Dunwoody and E. L. Swenson, The algebraic torus theorem, Invent. Math. 140 (2000), no. 3, 605-637. MR 2001d:20039

12. Michael Freedman, Joel Hass, and Peter Scott, Least area incompressible surfaces in 3manifolds, Invent. Math. 71 (1983), 609-642. MR 85e:57012

13. David Gabai, Convergence groups are Fuchsian groups, Annals of Math. 136 (1992), 447-510. MR 93m:20065

14. Wolfgang Haken, Theorie der Normalflächen, Acta Math. 105 (1961), 245-375. MR 25:4519a 
15. John Hempel, 3-manifolds, Ann. of Math. Studies, vol. 86, Princeton University Press, Princeton, New Jersey, 1976. MR 54:3702

16. William Jaco, Lectures on three-manifold topology, CBMS Lecture Notes, vol. 43, American Mathematical Society, Providence, RI, 1980. MR 81k:57009

17. William Jaco and J. Hyam Rubinstein, PL minimal surfaces in 3-manifolds, J. Differential Geom. 27 (1988), no. 3, 493-524. MR 89e:57009

18. William H. Jaco and Peter B. Shalen, Seifert fibered spaces in 3-manifolds, Memoirs of the American Mathematical Society, no. 220, American Mathematical Society, Providence, RI, September 1979. MR 81c:57010

19. Klaus Johannson, Homotopy equivalences of 3-manifolds with boundary, Lecture Notes in Mathematics, vol. 761, Springer-Verlag, Berlin, 1979. MR 82c:57005

20. H. Kneser, Geschlossene Flächen in dreidimensionalen Mannigfaltigkeiten, Jber. Deutsch. Math.-Verein. 38 (1929), 248-260.

21. Slawomir Kwasik and Reinhard Schultz, Icosahedral group actions on $\mathbf{R}^{3}$, Invent. Math. 108 (1992), no. 2, 385-402. MR 93b:57040

22. Sylvain Maillot, Quasi-isometries of groups, graphs and surfaces, Comment. Math. Helv. 76 (2001), no. 1, 29-60. MR 2002e:20078

23. William H. Meeks, III and Peter Scott, Finite group actions on 3-manifolds, Invent. Math. 86 (1986), no. 2, 287-346. MR 88b:57039

24. William H. Meeks, III and Shing-Tung Yau, Group actions on $\mathbf{R}^{3}$, The Smith conjecture (New York, 1979), Academic Press, Orlando, FL, 1984, pp. 167-179. MR 86i:57002

25. William H. Meeks, III, Leon Simon, and Shing-Tung Yau, Embedded minimal surfaces, exotic spheres, and manifolds with positive Ricci curvature, Annals of Mathematics 116 (1982), 621-659. MR 84f:53053

26. Geoffrey Mess, The Seifert conjecture and groups which are coarse quasiisometric to planes, Preprint.

27. Peter Scott, Compact submanifolds of 3-manifolds, J. London Math. Soc. (2) 7 (1973), 246250. MR 48:5080

28. Peter Scott, Ends of pairs of groups, J. Pure Appl. Algebra 11 (1977/78), no. 1-3, 179-198. MR 81h:20047

29. Peter Scott, There are no fake Seifert fibered spaces with infinite $\pi_{1}$, Annals of Math. 117 (1983), 35-70. MR 84c:57008

30. Peter Scott and Thomas Tucker, Some examples of exotic noncompact 3-manifolds, Quart. J. Math. Oxford Ser. (2) 40 (1989), no. 160, 481-499. MR 91b:57021

31. Yoshihiro Takeuchi, Partial solutions of the bad orbifold conjecture, Topology Appl. 72 (1996), no. 2, 113-120. MR 98g:57020

32. Yoshihiro Takeuchi and Misako Yokoyama, The geometric realizations of the decompositions of 3-orbifold fundamental groups, Topology Appl. 95 (1999), no. 2, 129-153. MR 2000g:57028

33. _ PL-least area 2-orbifolds and its applications to 3-orbifolds, Kyushu J. Math. 55 (2001), no. 1, 19-61. MR 2002k:57041

34. William P. Thurston, The geometry and topology of three-manifolds, Lecture notes, Princeton University (1976-1979).

35. _ Three dimensional manifolds, Kleinian groups and hyperbolic geometry, Bull. Amer. Math. Soc. 6 (1982), no. 3, 357-381. MR 83h:57019

36. _ Three-manifolds with symmetry, Preprint, 1982.

37. Pekka Tukia, Homeomorphic conjugates of Fuchsian groups, J. Reine Angew. Math. 391 (1988), 1-54. MR 89m:30047

38. Friedhelm Waldhausen, Eine Klasse von 3-dimensionalen Mannigfaltigkeiten, Invent. Math. 3-4 (1967), 308-333, 87-117. MR 38:3880

39. _ Gruppen mit Zentrum und 3-dimensionale Mannigfaltigkeiten, Topology 6 (1967), 505-517. MR 38:5223

40. 56-88. MR 36:7146

Département de Mathématiques, Université du Québec à Montréal, Case postale 8888 succursale centre-ville, Montréal, Canada H3C 3P8

E-mail address: maillot@math.uqam.ca 